\title{
MULTIPLE TUNNEL EFFECT FOR DISPERSIVE WAVES ON A STAR-SHAPED NETWORK: AN EXPLICIT FORMULA FOR THE SPECTRAL REPRESENTATION
}

\author{
F. ALI MEHMETI, R. HALLER-DINTELMANN, AND V. RÉGNIER
}

\begin{abstract}
We consider the Klein-Gordon equation on a star-shaped network composed of $n$ half-axes connected at their origins. We add a potential which is constant but different on each branch. The corresponding spatial operator is self-adjoint and we state explicit expressions for its resolvent and its resolution of the identity in terms of generalized eigenfunctions. This leads to a generalized Fourier type inversion formula in terms of an expansion in generalized eigenfunctions. Further we prove the surjectivity of the associated transformation, thus showing that it is in fact a spectral representation.

The characteristics of the problem are marked by the non-manifold character of the starshaped domain. Therefore the approach via the Sturm-Liouville theory for systems is not well-suited. The considerable effort to construct explicit formulas involving the tunnel effect generalized eigenfunctions is justified for example by the perspective to study the influence of tunnel effect on the $L^{\infty}$-time decay.
\end{abstract}

\section{INTRODUCTION}

This paper is motivated by the attempt to study the local behavior of waves near a node in a network of one-dimensional media having different dispersion properties. This leads to the study of a star-shaped network with semi-infinite branches. Results in experimental physics [18, 20], theoretical physics [15] and functional analysis [8, 14] describe new phenomena created in this situation by the dynamics of the tunnel effect: the delayed reflection and advanced transmission near nodes issuing two branches. It is of major importance for the comprehension of the vibrations of networks to understand these phenomena near ramification nodes i.e. nodes with at least 3 branches. The associated spectral theory induces a considerable complexity (as compared with the case of two branches) which is unraveled in the present paper.

Perturbation arguments do not seem to be suited for the above mentioned applications, as they lead to Neumann series in the solution formulae that hide the interesting effects.

The dynamical problem can be described as follows:

Let $N_{1}, \ldots, N_{n}$ be $n$ disjoint copies of $(0,+\infty)(n \geq 2)$. Consider numbers $a_{k}, c_{k}$ satisfying $0<c_{k}$, for $k=1, \ldots, n$ and $0 \leq a_{1} \leq a_{2} \leq \ldots \leq a_{n}<+\infty$. Find a vector $\left(u_{1}, \ldots, u_{n}\right)$ of functions $u_{k}:[0,+\infty) \times \overline{N_{k}} \rightarrow \mathbb{C}$ satisfying the Klein-Gordon equations

$$
\left[\partial_{t}^{2}-c_{k} \partial_{x}^{2}+a_{k}\right] u_{k}(t, x)=0, k=1, \ldots, n,
$$

on $N_{1}, \ldots, N_{n}$ coupled at zero by usual Kirchhoff conditions and complemented with initial conditions for the functions $u_{k}$ and their derivatives.

2000 Mathematics Subject Classification. Primary 34B45; Secondary 42A38, 47A10, 47A60, 47A70.

Key words and phrases. networks, spectral theory, resolvent, generalized eigenfunctions, functional calculus, evolution equations, dynamics of the tunnel effect, Klein-Gordon equation.

Parts of this work were done, while the second author visited the University of Valenciennes. He wishes to express his gratitude to F. Ali Mehmeti and the LAMAV for their hospitality. 
Reformulating this as an abstract Cauchy problem, one is confronted with the self-adjoint operator $A=\left(-c_{k} \cdot \partial_{x}^{2}+a_{k}\right)_{k=1, \ldots, n}$ in $L^{2}(N)$, with a domain that incorporates the Kirchhoff transmission conditions at zero. For an exact definition of $A$, we refer to Section 2 ,

Invoking functional calculus for this operator, the solution can be given in terms of

$$
e^{ \pm i \sqrt{A} t} u_{0} \text { and } e^{ \pm i \sqrt{A} t} v_{0}
$$

The refined study of transient phenomena thus requires concrete formulae for the spectral representation of $A$. The seemingly straightforward idea to view this task as a Sturm-Liouville problem for a system (following [27]) is not well-suited, because the resulting expansion formulae do not take into account the non-manifold character of the star-shaped domain. The ansatz used in [27] inhibits the exclusive use of generalized eigenfunctions satisfying the Kirchhoff conditions. This is proved in Theorem 8.1 in the appendix of this paper, which furnishes the comparison of the two approaches.

A first attempt to use well-suited generalized eigenfunctions in the ramified case but without tunnel effect [5] leads to a transformation whose inverse formula is different on each branch. The desired results for two branches but with tunnel effect are implicitly included in [27]. For $n$ branches but with the same $c_{k}$ and $a_{k}$ on all branches a variant of the above problem has been treated in [7] using Laplace transform in $t$.

In the present paper we start by following the lines of [5]. In Section [3, we define $n$ families of generalized eigenfunctions of $A$, i.e. formal solutions $F_{\lambda}^{k}$ for $\lambda \in\left[a_{1},+\infty\right)$ of the equation

$$
A F_{\lambda}^{k}=\lambda F_{\lambda}^{k}
$$

satisfying the Kirchhoff conditions in zero, such that $e^{ \pm i \sqrt{\lambda} t} F_{\lambda}^{k}(x)$ represent incoming or outgoing plane waves on all branches except $N_{k}$ for $\lambda \in\left[a_{n},+\infty\right)$. For $\lambda \in\left[a_{p}, a_{p+1}\right), 1 \leq p<n$ we have no propagation but exponential decay in $n-p$ branches: this expresses what we call the multiple tunnel effect, which is new with respect to [5]. Using variation of constants, we derive a formula for the kernel of the resolvent of $A$ in terms of the $F_{\lambda}^{k}$.

Following the classical procedure, in Section 4 we derive a limiting absorption principle for $A$, and then we insert $A$ in Stone's formula to obtain a representation of the resolution of the identity of $A$ in terms of the generalized eigenfunctions.

The aim of the paper, attained in Section 7, is the analysis of the Fourier type transformation

$$
(V f)(\lambda):=\left((V f)_{k}(\lambda)\right)_{k=1, \ldots, n}:=\left(\int_{N} f(x) \overline{\left(F_{\lambda}^{k}\right)}(x) d x\right)_{k=1, \ldots, n}
$$

in view of constructing its inverse. We show that it diagonalizes $A$ and determine a metric setting in which it is an isometry. This permits to express regularity and compatibility of $f$ in terms of decay of $V f$.

A major task in this context is to overcome the cyclic structure that the cyclic nature of the $n$-star induces in the underlying resolvent formula derived in Section 3. To this end, we use a symmetrization procedure that is carried out in Section 5. It combines the expression for the resolution of the identity $E(a, b)$ found in Section 4 with an ansatz for an expansion in generalized eigenfunctions:

$$
f(x)=\int_{a}^{b} \sum_{l, m=1}^{n} q_{l m}(\lambda) F_{\lambda}^{l}(x)(V f)_{k}(\lambda) d \lambda .
$$

This creates a $\left(3 n^{2}+1\right) \times n^{2}$ linear system for the $q_{l m}$, whose solution leads to the result in Theorem 5.3 and to the Plancherel type formula. 
A direct approach to the same symmetrization problem, carried out in Section 6, yields a closed formula for the matrix $q$ based on $n \times n$ matrices. This approach is to a great extent independent of the special setting and is thus supposed to be generalizable.

In Section 7 the desired inversion formula as well as the Plancherel type theorem are stated. Finally the domains of the powers of $A$ are characterized using the decay properties of $V f$. We show that $V$ is an ordered spectral representation (see Definition XII.3.15, p. 1216 of [17]). The spectrum has $n$ layers and it is $p$-fold on the frequency band $\left[a_{p}, a_{p+1}\right)$. This reflects a kind of continuous Zeemann effect caused by the constant, semi infinite potentials on the branches $N_{j}$ given by the terms $a_{j} u_{j}$. On this frequency band the generalized eigenfunctions have an exponential decay on $n-p$ branches, expressing the multiple tunnel effect.

Our results are designed to serve as tools in applications concerning the dynamics of the tunnel effect at ramification nodes. In particular, we think of retarded reflection (following [8, 20]), advanced transmission at barriers (following [18, 15, 14]), $L^{\infty}$-time decay (following [2, 3]), the study of more general networks of wave guides (for example microwave networks [25]), causality and global existence for nonlinear hyperbolic equations (following [9]) and the generalization to coupled transmission conditions (following [12]).

Finally, let us comment on some related results. The existing general literature on expansions in generalized eigenfunctions ([11, 24, 27] for example) does not seem to be helpful for our kind of problem: their constructions start from an abstractly given spectral representation. But in concrete cases you do not have an explicit formula for it at the beginning.

In [10] the relation of the eigenvalues of the Laplacian in an $L^{\infty}$-setting on infinite, locally finite networks to the adjacency operator of the network is studied. The question of the completeness of the corresponding eigenfunctions, viewed as generalized eigenfunctions in an $L^{2}$-setting, seems to be open.

In [22], the authors consider general networks with semi-infinite ends. They give a construction to compute generalized eigenfunctions from the coefficients of the transmission conditions and the asymptotic behaviour of eigenvalues is studied. The generality of the aproach, however, does not allow for explicit inversion formulas for a given family of generalized eigenfunctions.

Spectral theory for the Laplacian on finite networks has been studied since the 1980ies for example by J.P. Roth, J.v. Below, S. Nicaise, F. Ali Mehmeti. A list of references can be found in 1 .

In [23] the transport operator is considered on finite networks. The connection between the spectrum of the adjacency matrix of the network and the (discrete) spectrum of the transport operator is established. A generalization to infinite networks is contained in [16].

For surveys on results on networks and multistructures, cf. [4, 19].

Many results have been obtained in spectral theory for elliptic operators on various types of unbounded structures for example [21, 13, 6, 3], cf. especially the references mentioned in [3].

\section{DATA AND FUNCTIONAL ANALYTIC FRAMEWORK}

Let us introduce some notation which will be used throughout the rest of the paper.

Domain and functions. Let $N_{1}, \ldots, N_{n}$ be $n$ disjoint sets identified with $(0,+\infty)(n \in \mathbb{N}$, $n \geq 2$ ) and put $N:=\bigcup_{k=1}^{n} \overline{N_{k}}$, identifying the endpoints 0 , see [1] for a detailed definition. Furthermore, we write $[a, b]_{N_{k}}$ for the interval $[a, b]$ in the branch $N_{k}$. For the notation of functions two viewpoints are used:

- functions $f$ on the object $N$ and $f_{k}$ is the restriction of $f$ to $N_{k}$.

- $n$-tuples of functions on the branches $N_{k}$; then sometimes we write $f=\left(f_{1}, \ldots, f_{n}\right)$. 


\section{Transmission conditions.}

$$
\left(T_{0}\right):\left(u_{k}\right)_{k=1, \ldots, n} \in \prod_{k=1}^{n} C^{0}\left(\overline{N_{k}}\right) \text { satisfies } u_{i}(0)=u_{k}(0) \forall i, k \in\{1, \ldots, n\} .
$$

This condition in particular implies that $\left(u_{k}\right)_{k=1, \ldots, n}$ may be viewed as a well-defined function on $N$.

$$
\left(T_{1}\right):\left(u_{k}\right)_{k=1, \ldots, n} \in \prod_{k=1}^{n} C^{1}\left(\overline{N_{k}}\right) \text { satisfies } \sum_{k=1}^{n} c_{k} \cdot \partial_{x} u_{k}\left(0^{+}\right)=0 .
$$

Definition of the operator. Define the real Hilbert space $H=\prod_{k=1}^{n} L^{2}\left(N_{k}\right)$ with scalar product

$$
(u, v)_{H}=\sum_{k=1}^{n}\left(u_{k}, v_{k}\right)_{L^{2}\left(N_{k}\right)}
$$

and the operator $A: D(A) \longrightarrow H$ by

$$
\begin{aligned}
D(A) & =\left\{\left(u_{k}\right)_{k=1, \ldots, n} \in \prod_{k=1}^{n} H^{2}\left(N_{k}\right):\left(u_{k}\right)_{k=1, \ldots, n} \text { satisfies }\left(T_{0}\right) \text { and }\left(T_{1}\right)\right\}, \\
A\left(\left(u_{k}\right)_{k=1, \ldots, n}\right) & =\left(A_{k} u_{k}\right)_{k=1, \ldots, n}=\left(-c_{k} \cdot \partial_{x}^{2} u_{k}+a_{k} u_{k}\right)_{k=1, \ldots, n} .
\end{aligned}
$$

Note that, if $c_{k}=1$ and $a_{k}=0$ for every $k \in\{1, \ldots, n\}, A$ is the Laplacian in the sense of the existing literature, cf. [10, 22].

Proposition 2.1. The operator $A: D(A) \rightarrow H$ defined above is self-adjoint and satisfies $\sigma(A) \subset$ $\left[a_{1},+\infty\right)$.

Proof. Consider the Hilbert space

$$
V=\left\{\left(u_{k}\right)_{k=1, \ldots, n} \in \prod_{k=1}^{n} H^{1}\left(N_{k}\right):\left(u_{k}\right)_{k=1, \ldots, n} \text { satisfies }\left(T_{0}\right)\right\}
$$

with the canonical scalar product $(\cdot, \cdot)_{V}$. Then the bilinear form associated with $A+\left(\varepsilon-a_{1}\right) I$ is $a_{\varepsilon}: V \times V \rightarrow \mathbb{C}$ with

$$
a_{\varepsilon}(u, v)=\sum_{k=1}^{n}\left[c_{k}\left(\partial_{x} u_{k}, \partial_{x} v_{k}\right)_{L^{2}\left(N_{k}\right)}+\left(a_{k}+\varepsilon-a_{1}\right)\left(u_{k}, v_{k}\right)_{L^{2}\left(N_{k}\right)}\right] .
$$

Then clearly there is a $C>0$ with $a_{\varepsilon}(u, u) \geq C(u, u)_{V}$ for all $u \in V$ and all $\varepsilon>0$. By partial integration one shows that the Friedrichs extension of $\left(a_{\varepsilon}, V, H\right)$ is $\left(A+\left(\varepsilon-a_{1}\right) I, D(A)\right)$. Thus the operator $A+\left(\varepsilon-a_{1}\right) I$ is self-adjoint and positive. Hence $\sigma\left(A+\left(\varepsilon-a_{1}\right) I\right) \subset[0,+\infty)$ for all $\varepsilon>0$, what implies the assertion on the spectrum.

\section{EXPANSION IN GENERALIZED EIGENFUNCTIONS}

The aim of this section is to find an explicit expression for the kernel of the resolvent of the operator $A$ on the star-shaped network defined in the previous section.

Definition 3.1. An element $f \in \prod_{k=1}^{n} C^{\infty}\left(\overline{N_{k}}\right)$ is called generalized eigenfunction of $A$, if it satisfies $\left(T_{0}\right),\left(T_{1}\right)$ and formally the differential equation $A f=\lambda f$ for some $\lambda \in \mathbb{C}$. 
Lemma 3.2 (Green's formula on the star-shaped network). Denote by $V_{l_{1}, \ldots, l_{n}}$ the subset of the network $N$ defined by

$$
V_{l_{1}, \ldots, l_{n}}=\{0\} \cup \bigcup_{k=1}^{n}\left(0, l_{k}\right)_{N_{k}}
$$

Then $u, v \in D(A)$ implies

$$
\int_{V_{l_{1}, \ldots, l_{n}}} u^{\prime \prime}(x) v(x) d x=\int_{V_{l_{1}, \ldots, l_{n}}} u(x) v^{\prime \prime}(x) d x-\sum_{k=1}^{n} u\left(l_{k}\right) v^{\prime}\left(l_{k}\right)+\sum_{k=1}^{n} u^{\prime}\left(l_{k}\right) v\left(l_{k}\right) .
$$

Proof. Two successive integrations by parts are used and since both $u$ and $v$ belong to $D(A)$, they both satisfy the transmission conditions $\left(T_{0}\right)$ and $\left(T_{1}\right)$. So

Idem for $\sum_{k=1}^{n} u_{k}^{\prime}(0) v_{k}(0)$.

$$
\sum_{k=1}^{n} u_{k}(0) v_{k}^{\prime}(0)=u_{1}(0) \sum_{k=1}^{n} v_{k}^{\prime}(0)=0 \text {. }
$$

This Green formula yields now as usual an expression for the resolvent of $A$ in terms of the generalized eigenfunctions.

Proposition 3.3. Let $\lambda \in \rho(A)$ be fixed and let $e_{1}^{\lambda}$, $e_{2}^{\lambda}$ be generalized eigenfunctions of $A$, such that the Wronskian $w_{1,2}^{\lambda}(x)$ satisfies for every $x$ in $N$

$$
w_{1,2}^{\lambda}(x)=\operatorname{det} W\left(e_{1}^{\lambda}(x), e_{2}^{\lambda}(x)\right)=e_{1}^{\lambda}(x) \cdot\left(e_{2}^{\lambda}\right)^{\prime}(x)-\left(e_{1}^{\lambda}\right)^{\prime}(x) \cdot e_{2}^{\lambda}(x) \neq 0 .
$$

If for some $k \in\{1, \ldots, n\}$ we have $\left.e_{2}^{\lambda}\right|_{N_{k}} \in H^{2}\left(N_{k}\right)$ and $\left.e_{1}^{\lambda}\right|_{N_{m}} \in H^{2}\left(N_{m}\right)$ for all $m \neq k$, then for every $f \in H$ and $x \in N_{k}$

$$
[R(\lambda, A) f](x)=\frac{1}{c_{k} w_{1,2}^{\lambda}(x)} \cdot\left[\int_{(x,+\infty)_{N_{k}}} e_{1}^{\lambda}(x) e_{2}^{\lambda}\left(x^{\prime}\right) f\left(x^{\prime}\right) d x^{\prime}+\int_{N \backslash(x,+\infty)_{N_{k}}} e_{2}^{\lambda}(x) e_{1}^{\lambda}\left(x^{\prime}\right) f\left(x^{\prime}\right) d x^{\prime}\right] .
$$

Note that by the integral over $N$, we mean the sum of the integrals over $N_{k}, k=1, \ldots, n$.

Proof. Let $\lambda \in \rho(A)$. We shall show that the integral operator defined by the right-hand side of (11) is a left inverse of $\lambda I-A$. Let $u \in D(A)$ and $x \in N_{k}$. Then

$$
\begin{aligned}
I_{\lambda} & :=\int_{(x,+\infty)_{N_{k}}} e_{1}^{\lambda}(x) e_{2}^{\lambda}\left(x^{\prime}\right)(\lambda I-A) u\left(x^{\prime}\right) d x^{\prime}+\int_{N \backslash(x,+\infty)_{N_{k}}} e_{2}^{\lambda}(x) e_{1}^{\lambda}\left(x^{\prime}\right)(\lambda I-A) u\left(x^{\prime}\right) d x^{\prime} \\
& =e_{1}^{\lambda}(x) \lim _{l_{k} \rightarrow \infty} \int_{x}^{l_{k}} e_{2}^{\lambda}\left(x^{\prime}\right)(\lambda I-A) u\left(x^{\prime}\right) d x^{\prime}+e_{2}^{\lambda}(x) \lim _{l_{m} \rightarrow \infty, m \neq k} \int_{V_{l_{1}, \ldots, l_{k-1}, x, l_{k+1}, \ldots, l_{n}}} e_{1}^{\lambda}\left(x^{\prime}\right)(\lambda I-A) u\left(x^{\prime}\right) d x^{\prime},
\end{aligned}
$$

due to the dominated convergence Theorem, the integrands being in $L^{1}(\mathbb{R})$ by the hypotheses.

We have $u \in D(A) \subset \prod_{j=1}^{n} H^{2}\left(N_{j}\right)$ and

$$
\left.e_{2}^{\lambda}\right|_{N_{k}} \in H^{2}\left(N_{k}\right),\left.\quad e_{1}^{\lambda}\right|_{N_{m}} \in H^{2}\left(N_{m}\right), m \neq k
$$

by hypothesis and thus

$$
\begin{aligned}
\left.\left.\partial_{x} u\right|_{N_{k}}(x) \cdot e_{2}^{\lambda}\right|_{N_{k}}(x) \underset{x \rightarrow+\infty}{\longrightarrow} 0, & \left.\left.u\right|_{N_{k}}(x) \cdot \partial_{x} e_{2}^{\lambda}\right|_{N_{k}}(x) \underset{x \rightarrow+\infty}{\longrightarrow} 0, \\
\left.\left.\partial_{x} u\right|_{N_{m}}(x) \cdot e_{1}^{\lambda}\right|_{N_{m}}(x) \underset{x \rightarrow+\infty}{\longrightarrow} 0, & \left.\left.u\right|_{N_{m}}(x) \cdot \partial_{x} e_{1}^{\lambda}\right|_{N_{m}}(x) \underset{x \rightarrow+\infty}{\longrightarrow} 0, m \neq k,
\end{aligned}
$$

all products being in some $H^{2}\left(N_{j}\right)$. Recall that

$$
\int_{a}^{b} f^{\prime \prime} g=\int_{a}^{b} f g^{\prime \prime}-f(b) g^{\prime}(b)+f^{\prime}(b) g(b)+f(a) g^{\prime}(a)-f^{\prime}(a) g(a)
$$


for $f, g \in H^{2}((a, b))$. Now Lemma 3.2 and $(\lambda I-A) e_{r}^{\lambda}=0$ for $r=1,2$ imply

$$
\begin{aligned}
I_{\lambda}= & e_{1}^{\lambda}(x) \lim _{l_{k} \rightarrow \infty}\left[\int_{x}^{l_{k}}(\lambda I-A) e_{2}^{\lambda}\left(x^{\prime}\right) u\left(x^{\prime}\right) d x^{\prime}\right. \\
& \left.+c_{k}\left(-u\left(l_{k}\right) \partial_{x} e_{2}^{\lambda}\left(l_{k}\right)+\partial_{x} u\left(l_{k}\right) e_{2}^{\lambda}\left(l_{k}\right)+u(x) \partial_{x} e_{2}^{\lambda}(x)-\partial_{x} u(x) e_{2}^{\lambda}(x)\right)\right] \\
& +e_{2}^{\lambda}(x)\left[\lim _{l_{m} \rightarrow \infty, m \neq k} \int_{V_{l_{1}, \ldots, l_{k-1}, x, l_{k+1}, \ldots, l_{n}}}(\lambda I-A) e_{1}^{\lambda}\left(x^{\prime}\right) u\left(x^{\prime}\right) d x^{\prime}\right. \\
& \left.+\sum_{j \neq k} c_{j} \lim _{l_{j} \rightarrow \infty}\left(-u\left(l_{j}\right) \partial_{x} e_{1}^{\lambda}\left(l_{j}\right)+\partial_{x} u\left(l_{j}\right) e_{1}^{\lambda}\left(l_{j}\right)\right)+c_{k}\left(-u(x) \partial_{x} e_{1}^{\lambda}(x)+\partial_{x} u(x) e_{1}^{\lambda}(x)\right)\right] \\
= & c_{k}\left(e_{1}^{\lambda}(x) \partial_{x} e_{2}^{\lambda}(x)-\partial_{x} e_{1}^{\lambda}(x) e_{2}^{\lambda}(x)\right) u(x) \\
= & c_{k} w_{1,2}^{\lambda}(x) u(x) .
\end{aligned}
$$

Now the invertibility of $\lambda I-A$ implies the result.

Definition 3.4 (Generalized eigenfunctions of $A$ ). For $k \in\{1, \ldots, n\}$ and $\lambda \in \mathbb{C}$ let

$$
\xi_{k}(\lambda):=\sqrt{\frac{\lambda-a_{k}}{c_{k}}} \quad \text { and } \quad s_{k}:=-\frac{\sum_{l \neq k} c_{l} \xi_{l}(\lambda)}{c_{k} \xi_{k}(\lambda)} .
$$

Here, and in all what follows, the complex square root is chosen in such a way that $\sqrt{r \cdot e^{i \phi}}=$ $\sqrt{r} e^{i \phi / 2}$ with $r>0$ and $\phi \in[-\pi, \pi)$.

For $\lambda \in \mathbb{C}$ and $j, k \in\{1, \ldots, n\}, F_{\lambda}^{ \pm, j}: N \rightarrow \mathbb{C}$ is defined for $x \in \overline{N_{k}}$ by $F_{\lambda}^{ \pm, j}(x):=F_{\lambda, k}^{ \pm, j}(x)$ with

$$
\left\{\begin{array}{l}
F_{\lambda, j}^{ \pm, j}(x)=\cos \left(\xi_{j}(\lambda) x\right) \pm i s_{j}(\lambda) \sin \left(\xi_{j}(\lambda) x\right), \\
F_{\lambda, k}^{ \pm, j}(x)=\exp \left( \pm i \xi_{k}(\lambda) x\right), \quad \text { for } k \neq j .
\end{array}\right.
$$

Remark 3.5. - $F_{\lambda}^{ \pm, j}$ satisfies the transmission conditions $\left(T_{0}\right)$ and $\left(T_{1}\right)$ and formally it holds $A F_{\lambda}^{ \pm, j}=\lambda F_{\lambda}^{ \pm, j}$. Thus it is a generalized eigenfunction of $A$, but clearly $F_{\lambda}^{ \pm, j}$ does not belong to $H$, so it is not a classical eigenfunction.

- For $\operatorname{Im}(\lambda) \neq 0$, the function $F_{\lambda, k}^{ \pm, j}$, where the +-sign (respectively --sign) is chosen if $\operatorname{Im}(\lambda)>0$ (respectively $\operatorname{Im}(\lambda)<0$ ), belongs to $H^{2}\left(N_{k}\right)$ for $k \neq j$. This feature is used in the formula for the resolvent of $A$.

Definition 3.6 (Kernel of the resolvent). Let $w: \mathbb{C} \rightarrow \mathbb{C}$ be defined by $w(\lambda):= \pm i \cdot \sum_{j=1}^{n} c_{j} \xi_{j}(\lambda)$. For any $\lambda \in \mathbb{C}$ such that $w(\lambda) \neq 0, j \in\{1, \ldots, n\}$, and for every $x \in \overline{N_{j}}$, we define

$$
K\left(x, x^{\prime}, \lambda\right)=\left\{\begin{array}{l}
\frac{1}{w(\lambda)} F_{\lambda, j}^{ \pm, j}(x) F_{\lambda, j}^{ \pm, j+1}\left(x^{\prime}\right), \text { for } x^{\prime} \in \overline{N_{j}}, x^{\prime}>x, \\
\frac{1}{w(\lambda)} F_{\lambda, j}^{ \pm, j+1}(x) F_{\lambda}^{ \pm, j}\left(x^{\prime}\right), \text { for } x^{\prime} \in \overline{N_{k}}, k \neq j \text { or } x^{\prime} \in \overline{N_{j}}, x^{\prime}<x .
\end{array}\right.
$$

In the whole formula + (respectively - ) is chosen, if $\operatorname{Im}(\lambda)>0$ (respectively $\operatorname{Im}(\lambda) \leq 0$ ). Finally, the index $j$ is to be understood modulo $n$, that is to say, if $j=n$, then $j+1=1$.

Figure 1 shows the domain of the kernel $K(\cdot, \cdot, \lambda)$ in the case $n=3$ with its three main diagonals, where the kernel is not smooth. We will show in Theorem 3.8 that $K$ is indeed the kernel of the resolvent of $A$. In order to do so, we collect some useful observations in the following lemma. 


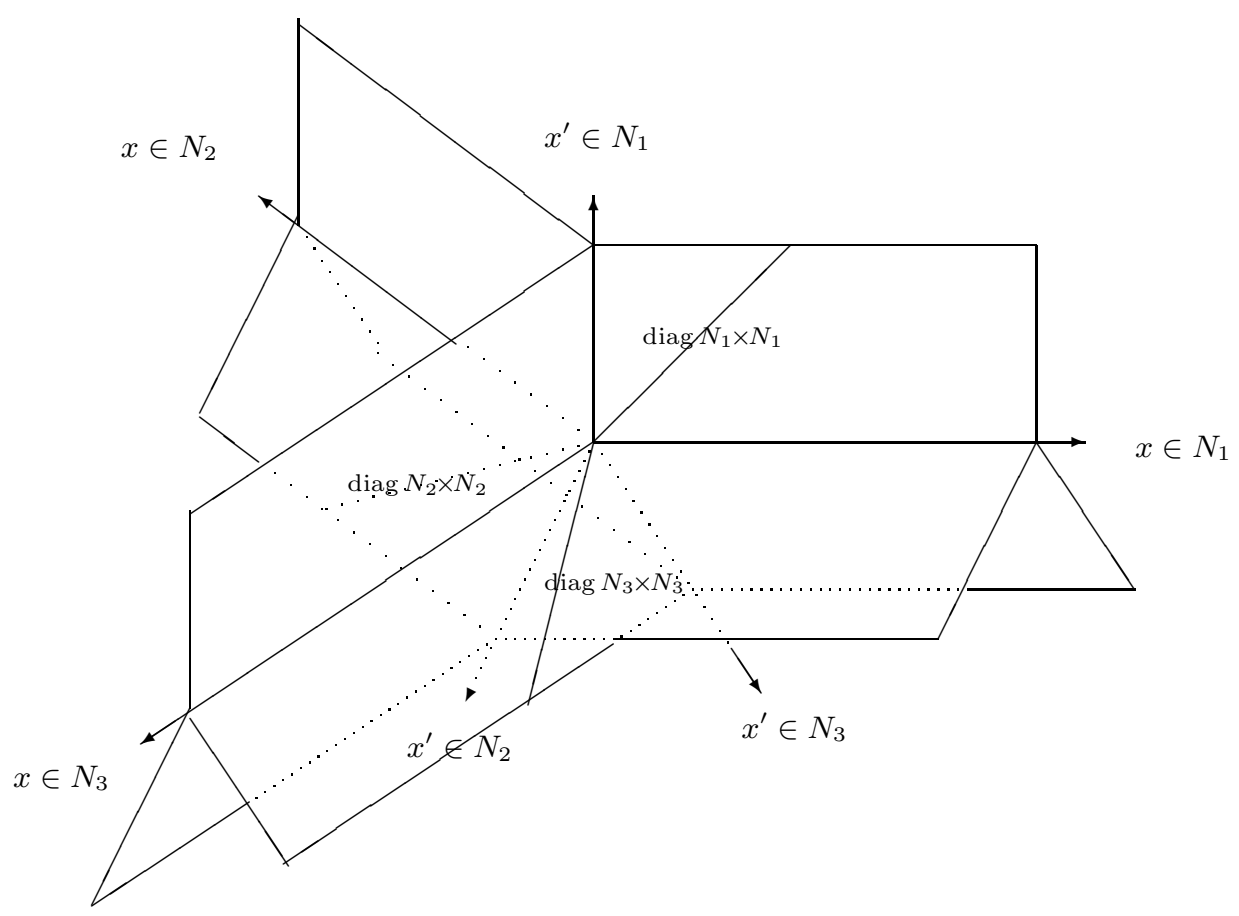

FiguRE $1 . N \times N$ in the case $n=3$

Note that in particular, if $c_{j}=c$ and $a_{j}=0$ for all $j \in\{1, \ldots, n\}$, then $w(\lambda)= \pm i n c \sqrt{\lambda}$ for all $j \in\{1, \ldots, n\}$, which only vanishes for $\lambda=0$. On the other hand, if there exist $i$ and $j$ in $\{1, \ldots, n\}$, such that $a_{i} \neq a_{j}$, then it is clear that $w(\lambda)$ never vanishes on $\mathbb{R}$, but we need to know, if it vanishes on $\mathbb{C}$.

Lemma 3.7. $\quad$ i) For $a_{1} \leq \lambda$ and $\varepsilon \geq 0$ holds $|w(\lambda-i \varepsilon)|^{2} \geq \sum_{j=1}^{n} c_{j}\left|\lambda-a_{j}\right|$.

ii) For $\lambda \in \rho(A)$ such that $\operatorname{Re}(\lambda) \geq a_{1}$, the Wronskian $w$ only vanishes at $\lambda=\alpha$, if $a_{k}=\alpha$ for all $k \in\{1, \ldots, n\}$.

Proof. We first prove ii). Note that for $z_{1}, z_{2}, \ldots, z_{n} \in \mathbb{C}$ holds

$$
\left|\sum_{j=1}^{n} z_{j}\right|^{2}=\sum_{j=1}^{n}\left|z_{j}\right|^{2}+2 \sum_{\substack{k, l=1 \\ k \neq l}}^{n} \operatorname{Re}\left(z_{k} \overline{z_{l}}\right) .
$$

With $z_{k}:=c_{k} \xi_{k}(\lambda-i \varepsilon)$ and the abbreviation $\eta_{k}:=\sqrt{\lambda-i \varepsilon-a_{k}}$ it follows

$$
|w(\lambda-i \varepsilon)|^{2}=\sum_{j=1}^{n} c_{j}\left|\eta_{j}\right|^{2}+2 \sum_{\substack{k, l=1 \\ k \neq l}}^{n} c_{k} c_{l} \operatorname{Re}\left(\eta_{k} \overline{\eta_{l}}\right) .
$$

Thus it suffices to show $\operatorname{Re}\left(\eta_{k} \bar{\eta}_{l}\right) \geq 0$ for $k, l=1, \ldots, n$ with $k \neq l$. With our convention $\sqrt{z}=\sqrt{|z|} e^{i \frac{\arg (z)}{2}}, \arg (z) \in[-\pi, \pi)$, this means

$$
\arg \left(\eta_{k} \overline{\eta_{l}}\right) \in\left[-\frac{\pi}{2}, \frac{\pi}{2}\right]
$$

Without loss of generality, let $k<l$. Then we have $a_{k} \leq a_{l}$ and there are three possible positions of $\lambda$ : 
- $a_{l} \leq \lambda$ :

Then for $r=k$ and for $r=l$ we have $\arg \left(\lambda-i \varepsilon-a_{r}\right) \in\left[-\frac{\pi}{2}, 0\right]$ and therefore

$$
\arg \left(\eta_{r}\right)=\frac{1}{2} \arg \left(\lambda-i \varepsilon-a_{r}\right) \in\left[-\frac{\pi}{4}, 0\right] .
$$

Using $\lambda-a_{k}>\lambda-a_{l}$, this implies

$$
\arg \left(\eta_{k} \overline{\eta_{l}}\right)=\arg \left(\eta_{k}\right)-\arg \left(\eta_{l}\right) \in\left[0, \frac{\pi}{4}\right] .
$$

- $a_{k} \leq \lambda<a_{l}$ :

Then $\arg \left(\lambda-i \varepsilon-a_{k}\right) \in\left[-\frac{\pi}{2}, 0\right]$ and therefore

$$
\arg \left(\eta_{k}\right)=\frac{1}{2} \arg \left(\lambda-i \varepsilon-a_{k}\right) \in\left[-\frac{\pi}{4}, 0\right] .
$$

Furthermore, $\arg \left(\lambda-i \varepsilon-a_{l}\right) \in\left[-\pi,-\frac{\pi}{2}\right]$, and therefore

$$
\arg \left(\eta_{l}\right)=\frac{1}{2} \arg \left(\lambda-i \varepsilon-a_{l}\right) \in\left[-\frac{\pi}{2},-\frac{\pi}{4}\right] .
$$

Putting everything together we get

$$
\arg \left(\eta_{k} \overline{\eta_{l}}\right)=\arg \left(\eta_{k}\right)-\arg \left(\eta_{l}\right) \in\left[0, \frac{\pi}{2}\right] .
$$

- $\lambda<a_{k}$ :

In this case we get, again for $r=k$ and $r=l, \arg \left(\lambda-i \varepsilon-a_{r}\right) \in\left[-\pi,-\frac{\pi}{2}\right]$ and therefore

This yields

$$
\arg \left(\eta_{r}\right)=\frac{1}{2} \arg \left(\lambda-i \varepsilon-a_{r}\right) \in\left[-\frac{\pi}{2},-\frac{\pi}{4}\right] .
$$

$$
\arg \left(\eta_{k} \bar{\eta}_{l}\right)=\arg \left(\eta_{k}\right)-\arg \left(\eta_{l}\right) \in\left[-\frac{\pi}{4}, \frac{\pi}{4}\right]
$$

Thus, in all three cases we have $\arg \left(\eta_{k} \overline{\eta_{l}}\right) \in\left[-\frac{\pi}{4}, \frac{\pi}{2}\right]$ and, hence, $\operatorname{Re}\left(\eta_{k} \overline{\eta_{l}}\right) \geq 0$ for all $k, l=$ $1, \ldots, n$ with $k \neq l$.

In order to prove lii), we note that the choice of the branch cut of the complex square root has been made in such a way that $\sqrt{\bar{\mu}}=\overline{\sqrt{\mu}}$ for all $\mu \in \mathbb{C}$. Thus $w(\bar{\mu})=\overline{w(\mu)}$ for all $\mu \in \mathbb{C}$. This implies that the first part of the lemma can be generalized to:

$$
|w(\mu)|^{2} \geq \sum_{j=1}^{n} c_{j}\left|\operatorname{Re}(\mu)-a_{j}\right|
$$

for every $\mu$ such that $\operatorname{Re}(\mu) \geq a_{1}$. Then $w(\mu)=0$ for such a $\mu$ implies $\left|\operatorname{Re}(\mu)-a_{j}\right|=0$ for all $j \in\{1, \ldots, n\}$. Thus all $a_{k}, k=1, \ldots, n$ have to be equal to some number $\alpha \geq 0$. Then $|w(\mu)|^{2}=\left(\sum_{k=1}^{n} \sqrt{c_{k}}\right)^{2}|\mu-\alpha|$, which only vanishes for $\mu=\alpha$.

Theorem 3.8. Let $f \in H$. Then, for $x \in N$ and $\lambda \in \rho(A)$ such that $\operatorname{Re}(\lambda) \geq a_{1}$

$$
[R(\lambda, A) f](x)=\int_{N} K\left(x, x^{\prime}, \lambda\right) f\left(x^{\prime}\right) d x^{\prime} .
$$

Proof. In (11), the generalized eigenfunction $e_{1}^{\lambda}$ can be chosen to be $F_{\lambda}^{ \pm, j}$. Then $e_{2}^{\lambda}$ can be $F_{\lambda}^{ \pm, l}$ with any $l \neq j$ so we have chosen $j+1$ to fix the formula. The choice has been done so that the integrands lie in $L^{1}(0,+\infty)$ (cf. the last item in Remark 3.5). Simple calculations yield the expression for the Wronskian $w(\lambda)$.

For $\lambda \in \rho(A)$ such that $\operatorname{Re}(\lambda) \geq a_{1}$, the Wronskian only vanishes at $\lambda=\alpha$ if $a_{k}=\alpha$ for all $k \in\{1, \ldots, n\}$ due to Lemma 3.7. But in this case, the Wronskian is $w(\lambda)=\left(\sum_{k=1}^{n} \sqrt{c_{k}}\right) \sqrt{\lambda-\alpha}$ and $w(\lambda)^{-1}$ has an $L^{1}$-singularity at $\lambda=\alpha$. 


\section{Applichtion of Stone's formula and limiting absorption principle}

Let us first recall Stone's formula (see Theorem XII.2.11 in [17]).

Theorem 4.1. Let $E$ be the resolution of the identity of a linear unbounded self-adjoint operator $T: D(T) \rightarrow H$ in a Hilbert space $H$ (i.e. $E(a, b)=\mathbf{1}_{(a, b)}(T)$ for $\left.(a, b) \in \mathbb{R}^{2}, a<b\right)$. Then, in the strong operator topology

$$
h(T) E(a, b)=\lim _{\delta \rightarrow 0^{+}} \lim _{\varepsilon \rightarrow 0^{+}} \frac{1}{2 \pi i} \int_{a+\delta}^{b-\delta} h(\lambda)[R(\lambda-\varepsilon i, T)-R(\lambda+\varepsilon i, T)] d \lambda
$$

for all $(a, b) \in \mathbb{R}^{2}$ with $a<b$ and for any continuous scalar function $h$ defined on the real line.

To apply this formula we need to study the behaviour of the resolvent $R(\lambda, A)$ for $\lambda$ approaching the spectrum of $A$. Lemma 3.7 will be useful as well as the following results. First we state an estimate that follows directly from the definition of $s_{j}$.

Lemma 4.2. Let $\delta>0$ be fixed. For all $a_{1} \leq \lambda, 0<\varepsilon<\delta$ and $j=1, \ldots, n$ holds

$$
\left|s_{j}(\lambda-i \varepsilon)\right| \leq M(\lambda, \delta):=\max _{j=1, \ldots, n}\left\{\frac{1}{\sqrt{\left|\lambda-a_{j}\right|}}\right\} \sum_{k=1}^{n}\left(\left(\lambda-a_{k}\right)^{2}+\delta^{2}\right)^{1 / 4} .
$$

Note that $M(\cdot, \delta) \in L_{\mathrm{loc}}^{1}\left(\left[a_{1},+\infty\right)\right)$. Furthermore, if $a_{1}=\ldots=a_{n}$, then

$$
s_{j}(\mu)=\frac{1}{\sqrt{c_{j}}} \sum_{\substack{k=1 \\ k \neq j}}^{n} \sqrt{c_{k}}
$$

for all $\mu \in \mathbb{C}$, which means that $s_{j}$ is constant and we may take

$$
M(\lambda, \delta):=\max _{j=1, \ldots, n}\left\{\frac{1}{\sqrt{c_{j}}} \sum_{\substack{k=1 \\ k \neq j}}^{n} \sqrt{c_{k}}\right\} .
$$

Theorem 4.3 (Limiting absorption principle for $A$ ). Let $\delta>0$ be fixed and let $M(\lambda, \delta)$ be defined as in Lemma 4.2. Then for all $a_{1} \leq \lambda, 0<\varepsilon<\delta$ and $\left(x, x^{\prime}\right) \in N^{2}$ we have

i) $\lim _{\alpha \rightarrow 0} K\left(x, x^{\prime}, \lambda-i \alpha\right)=K\left(x, x^{\prime}, \lambda\right)$,

ii) $\left|K\left(x, x^{\prime}, \lambda-i \varepsilon\right)\right| \leq N(\lambda, \delta) e^{\gamma\left(x+x^{\prime}\right)}$, where $N(\lambda, \delta):=\frac{1+M(\lambda, \delta)}{\left(\sum_{j=1}^{n} c_{j}\left|\lambda-a_{j}\right|\right)^{1 / 2}}$ and $\gamma:=\max _{j=1, \ldots, n}\left\{c_{j}^{-\frac{1}{2}}\right\} \max \left\{\left(\left(a_{n}-a_{1}\right)^{2}+\delta^{2}\right)^{\frac{1}{4}}, 1, \delta\right\}$.

Proof. i) The complex square root is, by definition, continuous on $\{z \in \mathbb{C}: \operatorname{Im}(z) \leq 0\}$ (cf. Definition 3.4), hence the continuity of $K\left(x, x^{\prime}, \cdot\right)$ from below on the real axis. Note that $x$ and $x^{\prime}$ are fixed parameters in this context.

ii) For $\mu=\lambda-i \varepsilon$ and $x \in \overline{N_{j}}$ we have in concrete terms

$$
K\left(x, x^{\prime}, \mu\right)=\frac{1}{w(\mu)} \begin{cases}{\left[\cos \left(\xi_{j}(\mu) x\right)-i s_{j}(\mu) \sin \left(\xi_{j}(\mu) x\right)\right] \exp \left(-i \xi_{j}(\mu) x^{\prime}\right),} & x^{\prime} \in \overline{N_{j}}, x^{\prime}>x, \\ \exp \left(-i \xi_{j}(\mu) x\right)\left[\cos \left(\xi_{j}(\mu) x^{\prime}\right)-i s_{j}(\mu) \sin \left(\xi_{j}(\mu) x^{\prime}\right)\right], & x^{\prime} \in \overline{N_{j}}, x^{\prime}<x, \\ \exp \left(-i \xi_{j}(\mu) x\right) \exp \left(-i \xi_{k}(\mu) x^{\prime}\right), & x^{\prime} \in \overline{N_{k}}, k \neq j .\end{cases}
$$

Now, let us first look at the case $\lambda>a_{n}$. Then

$$
\left|\exp \left(-i \xi_{j}(\mu) x\right)\right| \leq \exp \left(\left|\operatorname{Im}\left(\xi_{j}(\mu) x\right)\right|\right)=\exp \left(c_{j}^{-1 / 2}\left|\operatorname{Im}\left(\sqrt{\lambda-i \varepsilon-a_{j}}\right)\right|\right) .
$$

Using the fact, that for $z \in \mathbb{C}$ with $|\arg (z)| \leq \pi / 2$ we have $|\operatorname{Im}(\sqrt{z})| \leq \max \{1,|\operatorname{Im}(z)|\}$, we obtain

$$
\left|\exp \left(-i \xi_{j}(\mu) x\right)\right| \leq \exp \left(c_{j}^{-1 / 2} \max \{1, \delta\} x\right) .
$$


In the case $a_{1} \leq \lambda \leq a_{n}$ we find

$$
\left|\xi_{j}(\mu)\right|=\sqrt{\frac{\left|\mu-a_{j}\right|}{c_{j}}}=c_{j}^{-1 / 2}\left(\left(\lambda-a_{j}\right)^{2}+\varepsilon^{2}\right)^{1 / 4} \leq c_{j}^{-1 / 2}\left(\left(a_{n}-a_{j}\right)^{2}+\delta^{2}\right)^{1 / 4} .
$$

Using these estimates and $\left|e^{z}\right|=e^{\operatorname{Re}(z)} \leq e^{|z|}$, we find for all $\lambda \geq a_{1}$

$$
\begin{aligned}
\left|K\left(x, x^{\prime}, \mu\right)\right| & \leq \frac{1}{|w(\mu)|} \begin{cases}\left(1+\left|s_{j}(\mu)\right|\right) \exp \left(\left|\xi_{j}(\mu)\right| x\right) \exp \left(\left|\xi_{j}(\mu)\right| x^{\prime}\right), & x^{\prime} \in \overline{N_{j}}, \\
\exp \left(\left|\xi_{j}(\mu)\right| x\right) \exp \left(\left|\xi_{k}(\mu)\right| x^{\prime}\right), & x^{\prime} \in \overline{N_{k}}, k \neq j\end{cases} \\
& \leq \frac{1}{|w(\mu)|}\left(1+\left|s_{j}(\mu)\right|\right) \exp \left(\gamma\left(x+x^{\prime}\right)\right) .
\end{aligned}
$$

The conclusion now follows using Lemma 3.7 and Lemma 4.2 .

Note that these estimates in particular imply that $N(\cdot, \delta) \in L_{\text {loc }}^{1}\left(\left[a_{1},+\infty\right)\right)$. In fact, if $a_{1}=$ $\ldots=a_{n}$, then $M(\lambda, \delta)$ can be chosen to be constant, see Lemma 4.2, and the denominator of $N$ causes only an $L_{\text {loc }}^{1}$-type singularity. On the other hand, if there are two different $a_{j}$, the denominator of $N$ is never zero and $M(\cdot, \delta) \in L_{\mathrm{loc}}^{1}\left(\left[a_{1},+\infty\right)\right)$ again by Lemma 4.2.

Lemma 4.4. For $\left(x, x^{\prime}\right) \in N^{2}$ and $\lambda \in \mathbb{C}$, it holds $K\left(x, x^{\prime}, \bar{\lambda}\right)=\overline{K\left(x, x^{\prime}, \lambda\right)}$.

Proof. The choice of the branch cut of the complex square root has been made such that $\sqrt{\bar{\lambda}}=$ $\overline{\sqrt{\lambda}}$ for all $\lambda \in \mathbb{C}$. This implies $\overline{e^{i \sqrt{\lambda} x}}=e^{\overline{i \sqrt{\lambda} x}}=e^{-i \sqrt{\bar{\lambda}} x}$ for all $\lambda \in \mathbb{C}$ and $x \in \mathbb{R}$. Thus it holds

$$
\overline{F_{\lambda}^{+, j}(x)}=F_{\bar{\lambda}}^{-, j}(x) \text { and } \overline{F_{\lambda}^{-, j}(x)}=F_{\bar{\lambda}}^{+, j}(x)
$$

for all $\lambda \in \mathbb{C}, x \in N$ and $j \in\{1, \ldots, n\}$. In the same way we have $\overline{w(\lambda)}=-w(\bar{\lambda})$. Observe, that switching from $\lambda$ to $\bar{\lambda}$ the sign of the imaginary part is changing, so in the definition of $K\left(x, x^{\prime}, \lambda\right)$ we have to take the other sign, whenever there is a \pm -sign in the formula. This gives the assertion.

Now, we can deduce a first formula for the resolution of the identity of $A$.

Proposition 4.5. Take $f \in H=\prod_{j=1}^{n} L^{2}\left(N_{j}\right)$, vanishing almost everywhere outside a compact set $B \subset N$ and let $-\infty<a<b<+\infty$. Then for any continuous scalar function $h$ defined on the real line and for all $x \in N$

$$
\begin{aligned}
& (h(A) E(a, b) f)(x) \\
& =\int_{(a, b) \cap\left[a_{1},+\infty\right)} h(\lambda) \sum_{j=1}^{n} \mathbf{1}_{N_{j}}(x)\left\{\int _ { N } f ( x ^ { \prime } ) \left[\mathbf{1}_{(x,+\infty)_{N_{j}}}\left(x^{\prime}\right) \cdot \operatorname{Im}\left(\frac{1}{w(\lambda)} F_{\lambda}^{-, j}(x) F_{\lambda}^{-, j+1}\left(x^{\prime}\right)\right)\right.\right. \\
& \left.\left.\quad+\mathbf{1}_{N \backslash(x,+\infty)_{N_{j}}}\left(x^{\prime}\right) \cdot \operatorname{Im}\left(\frac{1}{w(\lambda)} F_{\lambda}^{-, j+1}(x) F_{\lambda}^{-, j}\left(x^{\prime}\right)\right)\right] d x^{\prime}\right\} d \lambda,
\end{aligned}
$$

where $E$ is the resolution of the identity of $A$ (cf. Theorem 4.1) and the index $j$ is to be understood modulo $n$, that is to say, if $j=n$, then $j+1=1$.

Proof. The proof is analogous to that of Lemma 3.13 in [2]. Let $g \in H$ be vanishing outside $B$. Then

$$
\begin{aligned}
& (h(A) E(a, b) f, g)_{H}=\left(\lim _{\delta \rightarrow 0^{+}} \lim _{\varepsilon \rightarrow 0^{+}} \frac{1}{2 \pi i} \int_{a+\delta}^{b-\delta} h(\lambda)[R(\lambda-\varepsilon i, A)-R(\lambda+\varepsilon i, A)] d \lambda f, g\right)_{H} \\
= & \lim _{\delta \rightarrow 0^{+}} \lim _{\varepsilon \rightarrow 0^{+}} \frac{1}{2 \pi i}\left(\int_{a+\delta}^{b-\delta} h(\lambda)[R(\lambda-\varepsilon i, A)-R(\lambda+\varepsilon i, A)] d \lambda f, g\right)_{H}
\end{aligned}
$$




$$
\begin{aligned}
& =\lim _{\delta \rightarrow 0^{+}} \lim _{\varepsilon \rightarrow 0^{+}} \frac{1}{2 \pi i} \int_{a+\delta}^{b-\delta} h(\lambda)([R(\lambda-\varepsilon i, A)-R(\lambda+\varepsilon i, A)] f, g)_{H} d \lambda \\
& =\lim _{\delta, \varepsilon \rightarrow 0^{+}} \frac{1}{2 \pi i} \int_{(a+\delta, b-\delta) \cap\left[a_{1},+\infty\right)} h(\lambda)\left(\int_{N} f\left(x^{\prime}\right)\left[K\left(\cdot, x^{\prime}, \lambda-i \varepsilon\right)-K\left(\cdot, x^{\prime}, \lambda+i \varepsilon\right)\right] d x^{\prime}, g(\cdot)\right)_{H} d \lambda \\
& =\lim _{\delta, \varepsilon \rightarrow 0^{+}} \frac{1}{2 \pi i} \int_{(a+\delta, b-\delta) \cap\left[a_{1},+\infty\right)} h(\lambda)\left(\int_{N} f\left(x^{\prime}\right)\left[K\left(\cdot, x^{\prime}, \lambda-i \varepsilon\right)-\overline{K\left(\cdot, x^{\prime}, \lambda-i \varepsilon\right)}\right] d x^{\prime}, g(\cdot)\right)_{H} d \lambda \\
& =\lim _{\delta, \varepsilon \rightarrow 0^{+}} \frac{1}{2 \pi i} \int_{(a+\delta, b-\delta) \cap\left[a_{1},+\infty\right)} h(\lambda)\left(\int_{N} f\left(x^{\prime}\right) 2 i \operatorname{Im}\left(K\left(\cdot, x^{\prime}, \lambda-i \varepsilon\right)\right) d x^{\prime}, g(\cdot)\right)_{H} d \lambda \\
& =\lim _{\delta \rightarrow 0^{+}} \frac{1}{\pi} \int_{(a+\delta, b-\delta) \cap\left[a_{1},+\infty\right)} h(\lambda)\left(\int_{N} f\left(x^{\prime}\right)\left[\lim _{\varepsilon \rightarrow 0^{+}} \operatorname{Im}\left(K\left(\cdot, x^{\prime}, \lambda-i \varepsilon\right)\right)\right] d x^{\prime}, g(\cdot)\right)_{H} d \lambda \\
& =\left(\frac{1}{\pi} \int_{(a, b) \cap\left[a_{1},+\infty\right)} h(\lambda) \int_{N} f\left(x^{\prime}\right) \operatorname{Im}\left(K\left(\cdot, x^{\prime}, \lambda-i 0\right)\right) d x^{\prime} d \lambda, g(\cdot)\right)_{H} \\
& =\int_{N} \frac{1}{\pi} \int_{(a, b) \cap\left[a_{1},+\infty\right)} h(\lambda)\left\{\int _ { N } f ( x ^ { \prime } ) \operatorname { I m } \left[\frac { 1 } { w ( \lambda ) } \sum _ { j = 1 } ^ { n } \mathbf { 1 } _ { \overline { N _ { j } } } ( x ) \left(\mathbf{1}_{(x,+\infty)_{N_{j}}}\left(x^{\prime}\right) F_{\lambda}^{-, j}(x) F_{\lambda}^{-, j+1}\left(x^{\prime}\right)\right.\right.\right. \\
& \left.\left.\left.+\mathbf{1}_{N \backslash(x,+\infty)_{N_{j}}}\left(x^{\prime}\right) F_{\lambda}^{-, j+1}(x) F_{\lambda}^{-, j}\left(x^{\prime}\right)\right)\right] d x^{\prime}\right\} d \lambda g(x) d x .
\end{aligned}
$$

Here, the justifications for the equalities are the following:

(2): Stone's formula (Theorem 4.1).

(3): After applying the operator valued integral to $f$, the two limits are in $H$. So they commute with the scalar product in $H$.

(44): $(\cdot f, g)_{H}$ is a continuous linear form on $\mathcal{L}(H)$, and can therefore be commuted with the vector-valued integration. Note that $\lambda \mapsto R(\lambda, A)$ is continuous on the half-plane $\left\{\lambda \in \mathbb{C}: \operatorname{Re}(\lambda)<a_{1}\right\}$, since the resolvent is holomorphic outside the spectrum, cf. Proposition 2.1.

(15): Theorem 3.8 .

(6): Lemma 4.4.

(7): $z-\bar{z}=2 i \cdot \operatorname{Im} z \forall z \in \mathbb{C}$.

(8): Dominated convergence. Since supp $f, \operatorname{supp} g$ and $[a, b]$ are compact, we use the limiting absorption principle (Theorem 4.3).

(9): Fubini's Theorem.

(10): Definition 3.6.

The assertion follows, since $g$ was arbitrary with compact support.

The unpleasant point about the formula in the above proposition is the apparent cut along the diagonal $\left\{x=x^{\prime}\right\}$ expressed by the characteristic functions in the variable $x^{\prime}$. In fact, there is no discontinuity and the two integrals recombine with respect to $x^{\prime}$. This is a consequence of the next lemma that gives an explicit representation of the integrand above.

In the following, we use the convention

$$
a_{n+1}:=+\infty,
$$

in order to unify notation and we set

$$
\xi_{j}^{\prime}:=i \xi_{j}
$$


Lemma 4.6. Let $j, k \in\{1, \ldots, n\}$ and let $\lambda$ be fixed in $\left(a_{p}, a_{p+1}\right)$, with $p \in\{1, \ldots, n\}$. Then $\operatorname{Im}\left[\frac{1}{w}\left(F_{\lambda}^{-, j+1}\right)_{j}(x)\left(F_{\lambda}^{-, j}\right)_{k}\left(x^{\prime}\right)\right]$ is given by the following expressions, respectively:

- If $k \geq j>p$ or $j \geq k>p$ (Case (a))

$$
\operatorname{Im}\left(\frac{1}{w}\right) e^{-\xi_{j}^{\prime} x-\xi_{k}^{\prime} x^{\prime}}
$$

- If $j<k \leq p$ or $k<j \leq p$ (Case (b), $j \neq k$ )

$$
\begin{aligned}
& \operatorname{Im}\left(\frac{1}{w}\right) \cos \left(\xi_{j} x\right) \cos \left(\xi_{k} x^{\prime}\right)-\operatorname{Im}\left(\frac{1}{w}\right) \sin \left(\xi_{j} x\right) \sin \left(\xi_{k} x^{\prime}\right) \\
& \quad-\operatorname{Re}\left(\frac{1}{w}\right) \cos \left(\xi_{j} x\right) \sin \left(\xi_{k} x^{\prime}\right)-\operatorname{Re}\left(\frac{1}{w}\right) \sin \left(\xi_{j} x\right) \cos \left(\xi_{k} x^{\prime}\right),
\end{aligned}
$$

- If $j=k \leq p$ (Case (b), $j=k$ )

$$
\begin{aligned}
& \operatorname{Im}\left(\frac{1}{w}\right) \cos \left(\xi_{j} x\right) \cos \left(\xi_{k} x^{\prime}\right)-\operatorname{Im}\left(\frac{s_{k}}{w}\right) \sin \left(\xi_{j} x\right) \sin \left(\xi_{k} x^{\prime}\right) \\
& \quad-\operatorname{Re}\left(\frac{1}{w}\right) \cos \left(\xi_{j} x\right) \sin \left(\xi_{k} x^{\prime}\right)-\operatorname{Re}\left(\frac{1}{w}\right) \sin \left(\xi_{j} x\right) \cos \left(\xi_{k} x^{\prime}\right),
\end{aligned}
$$

- If $j \leq p<k$ (Case (c))

$$
\operatorname{Im}\left(\frac{1}{w}\right) e^{-\xi_{k}^{\prime} x^{\prime}} \cos \left(\xi_{j} x\right)+\operatorname{Im}\left(\frac{1}{i w}\right) e^{-\xi_{k}^{\prime} x^{\prime}} \sin \left(\xi_{j} x\right)
$$

- If $k \leq p<j$ (Case (d))

$$
\operatorname{Im}\left(\frac{1}{w}\right) e^{-\xi_{j}^{\prime} x} \cos \left(\xi_{k} x^{\prime}\right)-\operatorname{Im}\left(\frac{1}{i w}\right) e^{-\xi_{j}^{\prime} x} \sin \left(\xi_{k} x^{\prime}\right)
$$

Proof. Since $\lambda$ belongs to $\left(a_{p}, a_{p+1}\right), \xi_{j}(\lambda)$ is a real number, if and only if $j \leq p$. Otherwise, $\xi_{j}$ is a purely imaginary number and we have $\xi_{j}=-i \xi_{j}^{\prime}$.

Now, the proof is pure calculation, using the following expressions for the generalized eigenfunctions in the case $j \neq k$

$$
\begin{aligned}
& \operatorname{Re}\left(F_{\lambda}^{-, j}\right)_{k}(x)= \begin{cases}\cos \left(\xi_{k} x\right), & \text { if } \xi_{k} \in \mathbb{R}, \text { i.e. } k \leq p, \\
e^{-\xi_{k}^{\prime} x}, & \text { if } \xi_{k} \in i \mathbb{R}, \text { i.e. } k>p,\end{cases} \\
& \operatorname{Im}\left(F_{\lambda}^{-, j}\right)_{k}(x)= \begin{cases}-\sin \left(\xi_{k} x\right), & \text { if } \xi_{k} \in \mathbb{R}, \text { i.e. } k \leq p, \\
0, & \text { if } \xi_{k} \in i \mathbb{R}, \text { i.e. } k>p,\end{cases}
\end{aligned}
$$

and for $j=k$

$$
\begin{aligned}
& \operatorname{Re}\left(F_{\lambda}^{-, k}\right)_{k}(x)= \begin{cases}\cos \left(\xi_{k} x\right)+\operatorname{Im}\left(s_{k}\right) \sin \left(\xi_{k} x\right), & \text { if } \xi_{k} \in \mathbb{R}, \text { i.e. } k \leq p, \\
\operatorname{Re}\left(\frac{1}{2}\left(1+s_{k}\right)\right) e^{-\xi_{k}^{\prime} x}+\operatorname{Re}\left(\frac{1}{2}\left(1-s_{k}\right)\right) e^{\xi_{k}^{\prime} x}, & \text { if } \xi_{k} \in i \mathbb{R}, \text { i.e. } k>p,\end{cases} \\
& \operatorname{Im}\left(F_{\lambda}^{-, k}\right)_{k}(x)= \begin{cases}-\operatorname{Re}\left(s_{k}\right) \sin \left(\xi_{k} x\right), & \text { if } \xi_{k} \in \mathbb{R}, \text { i.e. } k \leq p, \\
\operatorname{Im}\left(\frac{1}{2}\left(1+s_{k}\right)\right) e^{-\xi_{k}^{\prime} x}+\operatorname{Im}\left(\frac{1}{2}\left(1-s_{k}\right)\right) e^{\xi_{k}^{\prime} x}, & \text { if } \xi_{k} \in i \mathbb{R}, \text { i.e. } k>p,\end{cases}
\end{aligned}
$$

respectively.

Theorem 4.7. Take $f \in H=\prod_{j=1}^{n} L^{2}\left(N_{j}\right)$, vanishing almost everywhere outside a compact set $B \subset N$ and let $-\infty<a<b<+\infty$. Then for any continuous scalar function $h$ defined on the real line and for all $x \in N$

$(h(A) E(a, b) f)(x)=\int_{[a, b] \cap\left[a_{1},+\infty\right)} h(\lambda) \sum_{j=1}^{n} \mathbf{1}_{N_{j}}(x)\left\{\int_{N} f\left(x^{\prime}\right) \operatorname{Im}\left[\frac{1}{w(\lambda)} F_{\lambda}^{-, j+1}(x) F_{\lambda}^{-, j}\left(x^{\prime}\right)\right] d x^{\prime}\right\} d \lambda$,

where again the index $j$ is to be understood modulo $n$, i.e, if $j=n$, then $j+1=1$. 
Proof. All the work has already been done. It remains to inspect the formulae for $j=k$ in the cases (a) and (b) of Lemma 4.6, to observe that the expressions are symmetric in $x$ and $x^{\prime}$. So for $x, x^{\prime} \in N_{j}$ with $x<x^{\prime}$ we find $F_{\lambda}^{-, j}(x) F_{\lambda}^{-, j+1}\left(x^{\prime}\right)=F_{\lambda}^{-, j}\left(x^{\prime}\right) F_{\lambda}^{-, j+1}(x)$, which implies the assertion.

\section{Symmetrization}

As was already explained in the introduction, the aim of this section will be to find complex numbers $q_{l, m}, l, m \in\{1, \ldots, n\}$, such that the resolution of identity of $A$ can be written as

$$
(E(a, b) f)(x)=\int_{a}^{b} \sum_{l, m=1}^{n} q_{l m}(\lambda) F_{\lambda}^{-, l}(x) \int_{N} \overline{F_{\lambda}^{-, m}}\left(x^{\prime}\right) f\left(x^{\prime}\right) d x^{\prime} d \lambda
$$

in order to eliminate the cyclic structure of the formula in Proposition 4.7.

In this section we shall often suppress the dependence on $\lambda$ of several quantities for the ease of notation, so $s_{j}=s_{j}(\lambda), q_{l, m}=q_{l, m}(\lambda), \xi_{j}=\xi_{j}(\lambda), \xi_{j}^{\prime}=\xi_{j}^{\prime}(\lambda)$ and $w=w(\lambda)$.

Lemma 5.1. Given $x \in N_{j}$, equation (12) is satisfied for all $a_{1} \leq a<b<+\infty$ and all $f \in L^{2}(N)$ with compact support, if and only if for all $j=1, \ldots, n$

$$
\operatorname{Im}\left[\frac{1}{w} F_{\lambda}^{-, j+1}(x) F_{\lambda}^{-, j}\left(x^{\prime}\right)\right]=\sum_{l, m=1}^{n} q_{l m}(\lambda) F_{\lambda}^{-, l}(x) \overline{F_{\lambda}^{-, m}}\left(x^{\prime}\right)
$$

for almost all $x^{\prime} \in N$ and $\lambda \geq a_{1}$. Here again the index $j$ has to be understood modulo $n$, that is to say, if $j=n$, then $j+1=1$.

Proof. If functions $q_{l, m}, l, m=1, \ldots, n$, satisfy (13) and if $a_{1} \leq a<b<+\infty$, we get by (11)

$$
\begin{aligned}
(E(a, b) f)(x) & =\int_{a}^{b} \sum_{j=1}^{n} \mathbf{1}_{N_{j}}(x)\left\{\int_{N} f\left(x^{\prime}\right) \sum_{l, m=1}^{n} q_{l m}(\lambda) F_{\lambda}^{-, l}(x) \overline{F_{\lambda}^{-, m}}\left(x^{\prime}\right) d x^{\prime}\right\} d \lambda \\
& =\int_{a}^{b} \sum_{l, m=1}^{n} q_{l m}(\lambda) F_{\lambda}^{-, l}(x) \int_{N} \overline{F_{\lambda}^{-, m}}\left(x^{\prime}\right) f\left(x^{\prime}\right) d x^{\prime} d \lambda
\end{aligned}
$$

which is (12).

For the converse implication, let (12) be satisfied for some $x \in N_{j}$ and all $a_{1} \leq a<b<+\infty$, as well as all $f \in L^{2}(N)$ with compact support. This means by (11)

$$
\int_{a}^{b} \int_{N} f\left(x^{\prime}\right) \operatorname{Im}\left[\frac{1}{w(\lambda)} F_{\lambda}^{-, j+1}(x) F_{\lambda}^{-, j}\left(x^{\prime}\right)\right] d x^{\prime} d \lambda=\int_{a}^{b} \sum_{l, m=1}^{n} q_{l m}(\lambda) F_{\lambda}^{-, l}(x) \int_{N} \overline{F_{\lambda}^{-, m}}\left(x^{\prime}\right) f\left(x^{\prime}\right) d x^{\prime} d \lambda .
$$

Firstly, we want to see that the integrands of the $\lambda$-integrals on both sides in fact have to be equal almost everywhere. In order to do so, we observe that they both are in $L^{1}\left(\left(a_{1},+\infty\right)\right)$ and use the following general observation: If $I$ is an interval and $g \in L^{1}(I)$ satisfies $\int_{J} g=0$ for all intervals $J \subseteq I$, then $g=0$ almost everywhere in $I$. Indeed, in this case we have for any Lebesgue point $x_{0} \in I$ of $g$ and every $\varepsilon>0$ (cf. [26. Theorem 8.8])

$$
\begin{aligned}
\left|g\left(x_{0}\right)\right| & =|\frac{1}{2 \varepsilon} \int_{x_{0}-\varepsilon}^{x_{0}+\varepsilon}\left(g\left(x_{0}\right)-g(x)\right) d x+\frac{1}{2 \varepsilon} \underbrace{\int_{x_{0}-\varepsilon}^{x_{0}+\varepsilon} g(x) d x}_{=0}| \\
& \leq \frac{1}{2 \varepsilon} \int_{x_{0}-\varepsilon}^{x_{0}+\varepsilon}\left|g(x)-g\left(x_{0}\right)\right| d x \longrightarrow 0 \quad(\varepsilon \rightarrow 0),
\end{aligned}
$$


which implies $g\left(x_{0}\right)=0$ for almost all $x_{0} \in I$.

This implies

$$
\int_{N} f\left(x^{\prime}\right) \operatorname{Im}\left[\frac{1}{w(\lambda)} F_{\lambda}^{-, j+1}(x) F_{\lambda}^{-, j}\left(x^{\prime}\right)\right] d x^{\prime}=\int_{N} \sum_{l, m=1}^{n} q_{l m}(\lambda) F_{\lambda}^{-, l}(x) \overline{F_{\lambda}^{-, m}}\left(x^{\prime}\right) f\left(x^{\prime}\right) d x^{\prime}
$$

for almost all $\lambda \geq a_{1}$ and all $f \in L^{2}(N)$ with compact support. By the fundamental theorem of variational calculus this implies the assertion.

In a next step, we explicitely write down equation (13) as a linear system for the values $q_{l m}$.

Lemma 5.2. The equation

$$
\operatorname{Im}\left[\frac{1}{w}\left(F_{\lambda}^{-, j+1}\right)_{j}(x)\left(F_{\lambda}^{-, j}\right)_{k}\left(x^{\prime}\right)\right]=\sum_{l, m=1}^{n} q_{l m}(\lambda)\left(F_{\lambda}^{-, l}\right)_{j}(x)\left(\overline{F_{\lambda}^{-, m}}\right)_{k}\left(x^{\prime}\right)
$$

holds for any $\left(x, x^{\prime}\right) \in N_{j} \times N_{k}$ and $\lambda \in\left(a_{p}, a_{p+1}\right)$, if and only if

- Case (a): if $k \geq j>p$ or if $j \geq k>p$

$$
\left\{\begin{aligned}
q_{j k} & =0 \\
\sum_{l \neq j} q_{l k} & =0 \\
\sum_{m \neq k} q_{j m} & =0 \\
\sum_{l \neq j, m \neq k} q_{l m} & =\operatorname{Im}\left[\frac{1}{w}\right]
\end{aligned}\right.
$$

- Case (b), $j \neq k$ : if $j<k \leq p$ or if $k<j \leq p$

$$
\left\{\begin{array}{lllll} 
& \sum_{l, m} q_{l m} & =\operatorname{Im}\left[\frac{1}{w}\right] \\
\sum_{l \neq j, m \neq k} q_{l m} & +\overline{s_{k}} \sum_{l \neq j} q_{l k} & +s_{j} \sum_{m \neq k} q_{j m} & +s_{j} \cdot \overline{s_{k}} \cdot q_{j k} & =-\operatorname{Im}\left[\frac{1}{w}\right] \\
\sum_{l \neq j, m \neq k} q_{l m} & +\overline{s_{k}} \sum_{l \neq j} q_{l k} & +\sum_{m \neq k} q_{j m} & +\overline{s_{k}} \cdot q_{j k} & =-i \cdot \operatorname{Im}\left[\frac{1}{i w}\right] \\
\sum_{l \neq j, m \neq k} q_{l m} & +\sum_{l \neq j} q_{l k} & +s_{j} \sum_{m \neq k} q_{j m} & +s_{j} \cdot q_{j k} & =i \cdot \operatorname{Im}\left[\frac{1}{i w}\right],
\end{array}\right.
$$

- Case (b), $j=k$ : if $j=k \leq p$

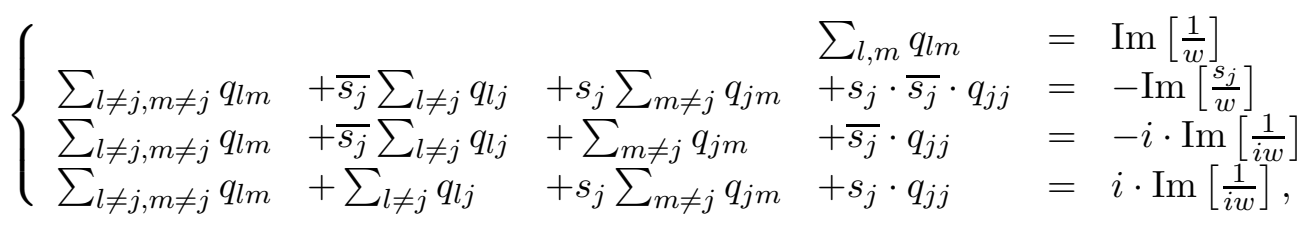

- Case (c): if $j \leq p<k$

$$
\left\{\begin{aligned}
q_{j k} & =0 \\
\sum_{l \neq j} q_{l k} & =0 \\
\sum_{m \neq k} q_{j m} & =\operatorname{Im}\left[\frac{1}{w}\right] \\
\sum_{l \neq j, m \neq k} q_{l m}+s_{j} \sum_{m \neq k} q_{j m} & =i \cdot \operatorname{Im}\left[\frac{1}{i w}\right]
\end{aligned}\right.
$$

- Case (d): if $k \leq p<j$

$$
\left\{\begin{aligned}
q_{j k} & =0 \\
\sum_{l \neq j} q_{l k} & =\operatorname{Im}\left[\frac{1}{w}\right] \\
\sum_{m \neq k} q_{j m} & =0 \\
\sum_{l \neq j, m \neq k} q_{l m}+\frac{s_{k}}{\sum_{l \neq j} q_{l k}} & =-i \cdot \operatorname{Im}\left[\frac{1}{i w}\right] .
\end{aligned}\right.
$$

Proof. The sum $\sum_{l, m=1}^{n} q_{l m}(\lambda)\left(F_{\lambda}^{-, l}\right)_{j}(x)\left(\overline{F_{\lambda}^{-, m}}\right)_{k}\left(x^{\prime}\right)$ is explicitely written in the different cases (a), (b), (c) and (d) as it was done in Lemma 4.6. Then the linear independence of the following families of functions is used to get the above systems for the $q_{l m}$ 's: 
- Case (a): $e^{A x+B x^{\prime}}, e^{A x-B x^{\prime}}, e^{-A x+B x^{\prime}}, e^{-A x-B x^{\prime}}$,

- Case (b): $\cos (A x) \cos \left(B x^{\prime}\right), \cos (A x) \sin \left(B x^{\prime}\right), \sin (A x) \cos \left(B x^{\prime}\right), \sin (A x) \sin \left(B x^{\prime}\right)$,

- Cases (c) and (d): $e^{A x} \cos \left(B x^{\prime}\right), e^{A x} \sin \left(B x^{\prime}\right), e^{-A x} \cos \left(B x^{\prime}\right), e^{-A x} \sin \left(B x^{\prime}\right)$,

where $A$ and $B$ are any fixed non-vanishing real numbers.

On the other hand: if the $q_{l m}$ satisfy the system indicated in the lemma, on both sides of the equation of the lemma we have the same linear combination of the functions given above. Thus equality holds.

Having the linear systems in the above lemma at hand, it remains to solve them. Indeed this is possible and we briefly indicate the necessary steps.

Firstly, if $\lambda<a_{1}$, we have $\xi_{j} \in i \mathbb{R}$ for all $j \in\{1, \ldots, n\}$. Thus, for all $j, k \in\{1, \ldots, n\}$ and all $\left(x, x^{\prime}\right) \in N_{j} \times N_{k}$

$$
\operatorname{Im}\left[\frac{1}{w}\left(F_{\lambda}^{-, j+1}\right)_{j}(x)\left(F_{\lambda}^{-, j}\right)_{k}\left(x^{\prime}\right)\right]=\operatorname{Im}\left(\frac{1}{w}\right) e^{-\xi_{j}^{\prime} x-\xi_{k}^{\prime} x^{\prime}}=0 .
$$

Now, let $\lambda$ be fixed in $\left(a_{p}, a_{p+1}\right)$, with $p \in\{1, \ldots, n\}$, remembering the convention $a_{n+1}=+\infty$. Due to the first equation of the corresponding system of cases (a), (c) and (d), the matrix $q:=\left(q_{l m}\right)_{l, m=1}^{n}$ has to be of the form

$$
q=\left(\begin{array}{c|c}
Q_{p} & 0 \\
\hline 0 & 0
\end{array}\right)
$$

with a $p \times p$ matrix $Q_{p}$.

Hence, the equations $\sum_{l \neq j} q_{l k}=0$ and $\sum_{m \neq k} q_{j m}=0$ in case (a) are obviously fulfilled, as well as $\sum_{l \neq j} q_{l k}=0$ in case (c) and $\sum_{m \neq k} q_{j m}=0$ in case (d). This means that only three equations remain from the cases (a), (c) and (d):

$$
\left\{\begin{aligned}
\sum_{l, m} q_{l m} & =\operatorname{Im}\left[\frac{1}{w}\right] \\
\sum_{m \neq k} q_{l m}+\overline{s_{k}} \sum q_{l k} & =-i \cdot \operatorname{Im}\left[\frac{1}{i w}\right] \\
\sum_{l \neq j} q_{l m}+s_{j} \sum q_{j m} & =i \cdot \operatorname{Im}\left[\frac{1}{i w}\right],
\end{aligned}\right.
$$

where in all the sums, $l$ and $m$ belong to $\{1, \ldots, p\}$. Now it is important to note that these three equations are already contained in the following system corresponding to the case (b), i.e. the only conditions for the $q_{l m}$ 's are the four following equations for a fixed $(j, k)$ such that $j<k \leq p$ or $k<j \leq p$ :

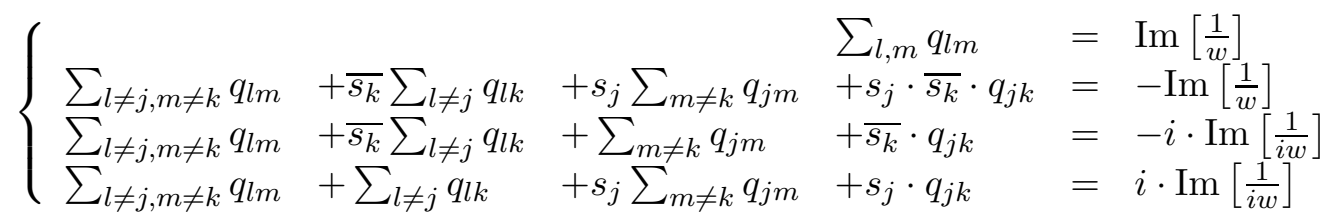

and, for $j=k \leq p$ :

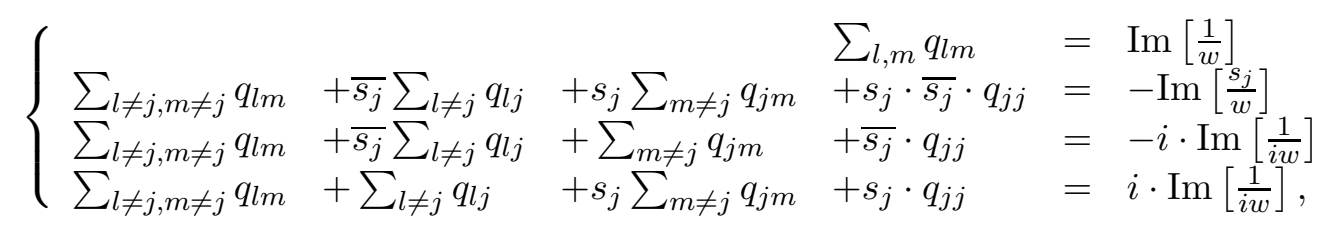

where, once more, $l$ and $m$ belong to $\{1, \ldots, p\}$ in all the sums. 
Now, we denote by $Q_{j k}:=\sum_{l \neq j} q_{l k}$ and $Q_{j k}^{\prime}:=\sum_{m \neq k} q_{j m}$. Using the fact that $\operatorname{Im}\left[\frac{1}{w}\right]=$ $\operatorname{Re}\left[\frac{1}{i w}\right]$, the above system (14) is

$$
\left\{\begin{array}{rrrl} 
& \sum_{l \neq j, m \neq k} q_{l m} & =\operatorname{Im}\left[\frac{1}{w}\right]-Q_{j k}-Q_{j k}^{\prime}-q_{j k} \\
\left(\overline{s_{k}}-1\right) Q_{j k}+ & \left(s_{j}-1\right) Q_{j k}^{\prime}+ & \left(s_{j} \overline{s_{k}}-1\right) q_{j k} & =-2 \operatorname{Re}\left[\frac{1}{i w}\right] \\
\left(\overline{s_{k}}-1\right) Q_{j k} & \left(s_{k}-1\right) q_{j k} & =-i \operatorname{Im}\left[\frac{1}{i w}\right]-\operatorname{Re}\left[\frac{1}{i w}\right]=-\frac{1}{i w} \\
& \left(s_{j}-1\right) Q_{j k}^{\prime}+ & \left(s_{j}-1\right) q_{j k} & =i \operatorname{Im}\left[\frac{1}{i w}\right]-\operatorname{Re}\left[\frac{1}{i w}\right]=\frac{1}{i \bar{w}} .
\end{array}\right.
$$

Since $s_{j}-1=-\frac{i w}{c_{j} \xi_{j}}$, the last three equations may be rewritten as

$$
\left\{\begin{array}{rrrr}
\left(-i c_{j} \xi_{j} \bar{w}\right) Q_{j k}+ & \left(i c_{k} \overline{\xi_{k}} w\right) Q_{j k}^{\prime}+\left(-i c_{j} \xi_{j} \bar{w}+i c_{k} \overline{\xi_{k}} w-|w|^{2}\right) q_{j k} & =\left(2 c_{j} c_{k} \xi_{j} \overline{\xi_{k}}\right) \operatorname{Re}\left[\frac{1}{i w}\right] \\
Q_{j k}+ & q_{j k} & =\frac{c_{k} \overline{\xi_{k}}}{|w|^{2}} \\
Q_{j k}^{\prime}+ & q_{j k} & =\frac{c_{j} \xi_{j}}{|w|^{2}}
\end{array}\right.
$$

and the Gauss method yields that $q_{j k}$ must vanish for $j \neq k$.

In the case $j=k$, we rewrite system (15) as above and the three equations to be solved turn out to be

$$
\left\{\begin{aligned}
\left(-i c_{j} \xi_{j} \bar{w}\right) Q_{j j}+ & \left(i c_{j} \overline{\xi_{j}} w\right) Q_{j j}^{\prime}+\left(-i c_{j} \xi_{j} \bar{w}+i c_{j} \overline{\xi_{j}} w-|w|^{2}\right) q_{j j} & =\left(2 c_{j}^{2} \xi_{j} \overline{\xi_{j}}\right)\left(\operatorname{Re}\left[\frac{1}{i w}\right]-\frac{1}{c_{j} \xi_{j}}\right) \\
Q_{j j}+ & q_{j j} & =\frac{c_{j} \overline{\xi_{j}}}{|w|^{2}} \\
Q_{j j}^{\prime}+ & q_{j j} & =\frac{c_{j} \xi_{j}}{|w|^{2}}
\end{aligned}\right.
$$

The Gauss method once more gives the only possible solution $q_{j j}=\frac{c_{j} \xi_{j}}{|w|^{2}}$ for any $j \in\{1, \ldots, p\}$.

With this candidate for a solution at hand, it is pure calculation to show that it is indeed a solution. Thus we have shown the following result.

Theorem 5.3. Let $A$ be defined as in Section 2 and the generalized eigenfunctions $F_{\lambda}^{-, j}$ be given by Definition 3.4. Take $f \in H=\prod_{j=1}^{n} L^{2}\left(N_{j}\right)$, vanishing almost everywhere outside a compact set $B \subset N$, and let $-\infty<a<b<+\infty$. Then for all $x \in N$

$$
(E(a, b) f)(x)=\int_{a}^{b} \sum_{l=1}^{n} q_{l}(\lambda) F_{\lambda}^{-, l}(x) \int_{N} \overline{F_{\lambda}^{-, l}}\left(x^{\prime}\right) f\left(x^{\prime}\right) d x^{\prime} d \lambda,
$$

where

$$
q_{l}(\lambda):= \begin{cases}0, & \text { if } \lambda<a_{l}, \\ \frac{c_{l}(\lambda) \xi_{l}(\lambda)}{|w(\lambda)|^{2}}, & \text { if } a_{l}<\lambda .\end{cases}
$$

Furthermore, for almost all $\lambda \in \mathbb{R}$ the matrix $q_{l, m}(\lambda)=\delta_{l m} \mathbf{1}_{\left(a_{l},+\infty\right)}(\lambda) c_{l}(\lambda) \xi_{l}(\lambda) /|w(\lambda)|^{2}$ is the unique matrix satisfying (12).

\section{A DIRECT APPROACH}

We have seen in the preceding section that a matrix $q(\lambda)$ satisfying (12) exists and is unique up to a null set. It is the aim of this section to deduce an alternative representation for $q(\lambda)$, involving only $n \times n$-matrices and not an $\left(3 n^{2}+1\right) \times n^{2}$ system as above. Since this approach is essentially independent of the special setting, it should be more convenient for generalizations.

In the following, let us consider the complex-valued generalized eigenfunctions $F_{\lambda}^{-, k}$ as functions on $N$ and not as elements of $\prod_{j=1}^{n} C^{\infty}\left(N_{j}\right)$. We introduce the notation 


$$
F_{\lambda}(x)=\left(\begin{array}{c}
F_{\lambda}^{-, 1}(x) \\
\vdots \\
F_{\lambda}^{-, n}(x)
\end{array}\right) .
$$

Denoting by $e_{k}=\left(\delta_{l k}\right)_{l=1, \ldots, n}=(0, \ldots, 0,1,0, \ldots, 0)^{T}$ the $k$-th unit vector in $\mathbb{C}^{n}$, we set $d_{1}(\lambda):=$ $F_{\lambda}(0)$ and for $j=2, \ldots, n$ we fix $x_{j} \in N_{j}$ and set $d_{j}(\lambda):=F_{\lambda}\left(x_{j}\right)$. Due to the form of our generalized eigenfunctions, we then have

$$
\begin{aligned}
& d_{1}(\lambda)=\sum_{k=1}^{n} e_{k}=(1, \ldots, 1)^{T} \\
& d_{j}(\lambda)=\beta_{j} e_{j}+\alpha_{j} \sum_{k \neq j} e_{k}=\left(\alpha_{j}, \ldots, \alpha_{j}, \beta_{j}, \alpha_{j}, \ldots, \alpha_{j}\right)^{T} \text { for } j=2, \ldots, n
\end{aligned}
$$

for suitable $\alpha_{j}, \beta_{j} \in \mathbb{C}$.

Using these vectors, we now define

$$
D(\lambda):=\sum_{j=1}^{n} d_{j}(\lambda) e_{j}^{T}
$$

Since $\alpha_{j} \neq \beta_{j}$, for every $j \in\{2, \ldots, n\}$, by construction, the matrix $D$ is invertible for any choice of $\left(x_{2}, \ldots, x_{n}\right)$ provided that $x_{j} \neq 0$ for all $j \in\{2, \ldots, n\}$. Indeed $\operatorname{det} D=\prod_{j=2}^{n}\left(\beta_{j}-\alpha_{j}\right)$.

Denoting by $C(\lambda)$ the diagonal matrix with $c_{11}=i$ and $c_{j j}=i \alpha_{j}$ for any $j \in\{2, \ldots, n\}$, we can formulate our theorem.

Theorem 6.1. The matrix $q(\lambda):=\left(q_{l m}(\lambda)\right)_{l, m=1, \ldots, n}$ satisfying (12) is given by

$$
\begin{aligned}
q(\lambda) & =\left(D(\lambda)^{-1}\right)^{T} \operatorname{Im}\left(\frac{1}{w(\lambda)} \sum_{j=1}^{n} e_{j} d_{j}(\lambda)^{T} e_{j+1} e_{j}^{T} D(\lambda)\right)\left(\overline{D(\lambda)}^{-1}\right)^{T} \\
& =\left(D(\lambda)^{-1}\right)^{T} \operatorname{Im}\left(\frac{-i}{w(\lambda)} C(\lambda) D(\lambda)\right)\left(\overline{D(\lambda)}^{-1}\right)^{T}
\end{aligned}
$$

for almost all $\lambda>a_{1}$. As previously, $j$ has to be understood modulo $n$, that is to say, $j+1=1$ if $j=n$.

Proof. By Lemma 5.1 the function $q$ satisfies (13). Using the matrices and vectors introduced above, for fixed $j \in\{1, \ldots, n\}$ this can be rewritten as

$$
\operatorname{Im}\left(\frac{1}{w(\lambda)} F_{\lambda}(x)^{T} e_{j+1} e_{j}^{T} F_{\lambda}\left(x^{\prime}\right)\right)=F_{\lambda}(x)^{T} q(\lambda) \overline{F_{\lambda}}\left(x^{\prime}\right)
$$

for $x \in N_{j}$ and $x^{\prime} \in N$.

Setting $x=x_{j}$ and $x^{\prime}=x_{k}$ we obtain by the definition of $d_{j}$

$$
\operatorname{Im}\left(\frac{1}{w(\lambda)} d_{j}(\lambda)^{T} e_{j+1} e_{j}^{T} d_{k}(\lambda)\right)=d_{j}(\lambda)^{T} q(\lambda) \overline{d_{k}(\lambda)}
$$

for all $j, k \in\{1, \ldots, n\}$ and thus

$$
\sum_{k=1}^{n} \sum_{j=1}^{n} e_{j} \operatorname{Im}\left(\frac{1}{w(\lambda)} d_{j}(\lambda)^{T} e_{j+1} e_{j}^{T} d_{k}(\lambda)\right) e_{k}^{T}=\sum_{k=1}^{n} \sum_{j=1}^{n} e_{j} d_{j}(\lambda)^{T} q(\lambda) \overline{d_{k}(\lambda)} e_{k}^{T}
$$


Using $\sum_{k=1}^{n} d_{k} e_{k}^{T}=D$, we obtain

$$
\operatorname{Im}\left(\frac{1}{w(\lambda)} \sum_{j=1}^{n} e_{j} d_{j}(\lambda)^{T} e_{j+1} e_{j}^{T} D\right)=D^{T} q \bar{D} .
$$

Since $D$ is invertible and the relation $\sum_{j=1}^{n} e_{j} d_{j}(\lambda)^{T} e_{j+1} e_{j}^{T}=-i C(\lambda)$ holds true, we now get the desired formula for $q$.

\section{A Plancherel-type Theorem}

In this section we prove a Plancherel type theorem for a Fourier type transformation $V$ and its inverse $V^{-1}$ associated with the generalized eigenfunctions $\left(F_{\lambda}^{-, k}\right)_{k=1, \ldots, n}$ introduced in Definition 3.4. Due to the fact that $V$ and $V^{-1}$ turn out to be not analogous, the surjectivity of $V$ will not follow from its injectivity and needs a separate proof.

Furthermore, we show that the fact that a function $u$ belongs to the space $D\left(A^{k}\right)$ can be formulated in terms of the decay rate of $V u$. These results should be useful for the resolution of evolution problems involving the spatial operator $A$ as well as for the analysis of the properties of a solution. For this part, we follow Section 4 of [2].

Definition 7.1. Let $q_{k}$ be the coefficients defined in Theorem 5.3. Considering for every $k=$ $1, \ldots, n$ the weighted $L^{2}$ space $L^{2}\left(\left(a_{k},+\infty\right), q_{k}\right)$, we set $L_{q}^{2}:=\prod_{k=1}^{n} L^{2}\left(\left(a_{k},+\infty\right), q_{k}\right)$. The corresponding scalar product is denoted by

$$
(F, G)_{q}:=\sum_{k=1}^{n} \int_{\left(a_{k},+\infty\right)} q_{k}(\lambda) F_{k}(\lambda) \overline{G_{k}(\lambda)} d \lambda
$$

and the norm by $|F|_{q}:=(F, F)_{q}^{1 / 2}$.

Note that $L_{q}^{2}$ is a Hilbert space, since it is the product of the Hilbert spaces $L^{2}\left(\left(a_{k},+\infty\right), q_{k}\right)$.

Now, we define the transformation $V$, together with its inverse $Z$, which is later on shown to diagonalize $A$.

Definition 7.2. i) For all $f \in L^{1}(N, \mathbb{C})$ and $k=1, \ldots, n$ the function $(V f)_{k}:\left[a_{k},+\infty\right) \rightarrow$ $\mathbb{C}$ is defined by

$$
(V f)_{k}(\lambda):=\int_{N} f(x) \overline{\left(F_{\lambda}^{-, k}\right)(x)} d x .
$$

ii) We choose a cut-off function $\chi \in C^{\infty}(\mathbb{R})$ such that $\chi \equiv 0$ on $\left(-\infty, a_{n}+1\right)$ and $\chi \equiv 1$ on $\left(a_{n}+2,+\infty\right)$. Then we set $X$ to be the space of all $\left(G_{1}, \ldots, G_{n}\right) \in L_{q}^{2}$, such that $G_{k} \in C^{\infty}\left(\left[a_{k},+\infty\right), \mathbb{C}\right)$ and $\chi G_{k}$ can be extended by zero to an element of $\mathcal{S}(\mathbb{R})$ for all $k \in\{1, \ldots, n\}$.

Now, for every $\left(G_{1}, \ldots, G_{n}\right) \in X$ we define $Z\left(G_{1}, \ldots, G_{n}\right): N \rightarrow \mathbb{C}$ by

$$
Z\left(G_{1}, \ldots, G_{n}\right)(x):=\sum_{k=1}^{n} \int_{\left(a_{k},+\infty\right)} q_{k}(\lambda) G_{k}(\lambda)\left(F_{\lambda}^{-, k}\right)(x) d \lambda .
$$

Lemma 7.3. Let $f \in L^{2}(N)$ and $G=\left(G_{1}, \ldots, G_{n}\right) \in X$. Then $G \in L_{q}^{2}, Z(G) \in L^{2}(N)=H$, $V f=\left((V f)_{1}, \ldots,(V f)_{n}\right) \in L_{q}^{2}$, and

$$
(G, V f)_{q}=(Z(G), f)_{H} .
$$


Proof. We have

$$
\begin{aligned}
& (G, V f)_{q}=\sum_{k=1}^{n}\left(G_{k},(V f)_{k}\right)_{L^{2}\left(\left(a_{k},+\infty\right), q_{k}\right)}=\sum_{k=1}^{n} \int_{\left(a_{k},+\infty\right)} q_{k}(\lambda) G_{k}(\lambda) \overline{(V f)_{k}}(\lambda) d \lambda \\
& =\sum_{k=1}^{n} \int_{\left(a_{k},+\infty\right)} q_{k}(\lambda) G_{k}(\lambda) \overline{\left(\int_{N} f(x) \overline{\left(F_{\lambda}^{-, k}\right)}(x) d x\right)} d \lambda \\
& =\int_{N}\left(\sum_{k=1}^{n} \int_{\left(a_{k},+\infty\right)} q_{k}(\lambda) G_{k}(\lambda)\left(F_{\lambda}^{-, k}\right)(x) d \lambda\right) \overline{f(x)} d x=(Z(G), f)_{H} .
\end{aligned}
$$

It only remains to make sure that the assumptions for Fubini's Theorem are satisfied to justify (16). In fact, it is sufficient to estimate $(\lambda, x) \mapsto q_{k}(\lambda)\left(F_{\lambda}^{-, k}\right)(x)$ on $\left(a_{k}, a_{k+1}\right) \times N$ for a fixed $k \in\{1, \ldots, n\}$, since $f$ is compactly supported and $G_{k}$ is rapidly decreasing.

In order to do so, recall that $c_{k} \xi_{k}(\lambda)=\sqrt{c_{k}\left(\lambda-a_{k}\right)}$ belongs to $\mathbb{R}$ if and only if $\lambda \geq a_{k}$ and to $i \mathbb{R}$ otherwise and that for any $\lambda>a_{1}$, we have $|w(\lambda)|^{2} \geq \sum_{l=1}^{n} c_{l}\left|\lambda-a_{l}\right|$ due to Lemma 3.7 . Thus, putting in the expression for $s_{k}(\lambda)$, cf. Definition [3.4, we get for $x \in N_{k}$

$$
\begin{aligned}
\left|q_{k}(\lambda)\left(F_{\lambda}^{-, k}\right)(x)\right| & =\left|\frac{c_{k} \xi_{k}(\lambda)}{|w(\lambda)|^{2}}\left(\cos \left(\xi_{k}(\lambda) x\right)-i s_{k}(\lambda) \sin \left(\xi_{k}(\lambda) x\right)\right)\right| \leq \frac{\left|c_{k} \xi_{k}(\lambda)\right|}{\sum_{l=1}^{n} c_{l}\left|\lambda-a_{l}\right|}\left(1+\left|s_{k}(\lambda)\right|\right) \\
& \leq \frac{\left|c_{k} \xi_{k}(\lambda)\right|}{\sum_{l=1}^{n} c_{l}\left|\lambda-a_{l}\right|} \cdot \frac{\sum_{l=1}^{n} c_{l}\left|\xi_{l}(\lambda)\right|}{\left|c_{k} \xi_{k}(\lambda)\right|} \leq \frac{\sum_{l=1}^{n} \sqrt{c_{l}} \sqrt{\left|\lambda-a_{l}\right|}}{\sum_{l=1}^{n} c_{l}\left|\lambda-a_{l}\right|}
\end{aligned}
$$

Furthermore, for $x \in N_{j}, j>k$, we have

$$
\left|q_{k}(\lambda)\left(F_{\lambda}^{-, k}\right)(x)\right|=\left|\frac{c_{k} \xi_{k}(\lambda)}{|w(\lambda)|^{2}} e^{-i \xi_{j}(\lambda) x}\right| \leq \frac{1}{\sqrt{c_{k}} \sqrt{\left|\lambda-a_{k}\right|}} \exp \left(-\sqrt{\frac{a_{j}-\lambda}{c_{j}}} x\right) .
$$

Finally, for $x \in N_{j}, j<k$,

$$
\left|q_{k}(\lambda)\left(F_{\lambda}^{-, k}\right)(x)\right|=\left|\frac{c_{k} \xi_{k}(\lambda)}{|w(\lambda)|^{2}} e^{-i \xi_{j}(\lambda) x}\right| \leq \frac{1}{\sqrt{c_{k}} \sqrt{\left|\lambda-a_{k}\right|}} .
$$

For all three cases the bound is an $L_{\text {loc }}^{1}$ function of $\lambda$, which is enough for the application of Fubini's Theorem, due to the properties of $f$ and $G$.

Lemma 7.4. Consider $f \in \prod_{k=1}^{n} C_{c}^{\infty}\left(N_{k}\right)$. Then $\left((V f)_{1}, \ldots,(V f)_{n}\right) \in X$.

Proof. As in Section 4 of [2], $(V f)_{k}$ is a linear combination of Fourier and Laplace transforms of functions in $C_{c}^{\infty}\left(N_{j}\right), j \in\{1, \ldots, n\}$.

The next step is to show that $V$ is an isometry and $Z$ is its right inverse. The proof of the following theorem is analogous to that of [2, Theorem 4.9].

Theorem 7.5. Endow $\prod_{k=1}^{n} C_{c}^{\infty}\left(N_{k}\right)$ with the norm of $H=\prod_{k=1}^{n} L^{2}\left(N_{k}\right)$. Then

i) $V: \prod_{k=1}^{n} C_{c}^{\infty}\left(N_{k}\right) \rightarrow L_{q}^{2}$ is isometric and can therefore be extended to an isometry $\tilde{V}: H \rightarrow L_{q}^{2}$.

In particular, for all $f \in H$ we have $|\tilde{V} f|_{q}^{2}=(f, f)_{H}$.

ii) $Z=\tilde{V}^{-1}$ on $\tilde{V}(H)$.

iii) $Z$ can be extended to a continuous operator on $L_{q}^{2}$. 
In the sequel we shall again write $V$ for $\tilde{V}$ for simplicity. Note that thanks to the density of $X$ in $L_{q}^{2}$, from Lemma 7.3 we get the Plancherel type formula

$$
(G, V f)_{q}=(Z(G), f)_{H} \quad \text { for all } f \in L^{2}(N) \text { and } G \in L_{q}^{2} \text {. }
$$

Set

$$
Y:=\left\{\left(G_{1}, \ldots, G_{n}\right) \in L_{q}^{2}: \operatorname{supp}\left(G_{j}\right) \text { compact and } \operatorname{supp}\left(G_{j}\right) \cap\left\{a_{1}, \ldots, a_{n}\right\}=\emptyset, j=1, \ldots, n\right\} .
$$

Then $Y$ is a dense subspace of $L_{q}^{2}$ and for our subsequent considerations it is important to know that also for all $G \in Y$ the transformation $Z$ is given by the formula from Definition 7.2 ii). This is the aim of the following lemma.

Lemma 7.6. Let $G \in Y$. Then

$$
Z(G)(x)=\sum_{k=1}^{n} \int_{\left(a_{k},+\infty\right)} q_{k}(\lambda) G_{k}(\lambda)\left(F_{\lambda}^{-, k}\right)(x) d \lambda .
$$

Proof. Let $f \in \prod_{k=1}^{n} C_{c}^{\infty}\left(N_{k}\right)$. Then, using (20), we get

$$
(Z(G), f)_{H}=(G, V f)_{q}=\sum_{k=1}^{n} \int_{\left(a_{k},+\infty\right)} G_{k}(\lambda) \int_{N} F_{\lambda}^{-, k}(x) f(x) d x q_{k}(\lambda) d \lambda .
$$

In order to apply Fubini's Theorem, we estimate for every $k=1, \ldots, n$

$$
\begin{aligned}
\int_{N}\left|G_{k}(\lambda) F_{\lambda}^{-, k}(x) f(x) q_{k}(\lambda)\right| d x & =\left|G_{k}(\lambda) q_{k}(\lambda)\right| \int_{N}\left|F_{\lambda}^{-, k}(x)\right||f(x)| d x \\
& \leq\left|G_{k}(\lambda)\right| \sup _{x \in \operatorname{supp}(f)}\left|q_{k}(\lambda) F_{\lambda}^{-, k}(x)\right| \cdot\|f\|_{L^{1}(N)} .
\end{aligned}
$$

Investing the estimates from (17) - (19), we can estimate this further by

$$
\leq C \frac{1}{\sqrt{\left|\lambda-a_{k}\right|}}\left|G_{k}(\lambda)\right| \cdot\|f\|_{L^{1}(N)},
$$

and since $G \in Y$ this function is in $L^{1}\left(\left(a_{k},+\infty\right)\right)$, so we may apply the Fubini Theorem and obtain

$$
\begin{aligned}
(Z(G), f)_{H} & =\int_{N} \sum_{k=1}^{n} \int_{\left(a_{k},+\infty\right)} q_{k}(\lambda) G_{k}(\lambda) F_{\lambda}^{-, k}(x) d \lambda f(x) d x \\
& =\left(\sum_{k=1}^{n} \int_{\left(a_{k},+\infty\right)} q_{k}(\lambda) G_{k}(\lambda) F_{\lambda}^{-, k} d \lambda, f\right)_{H} .
\end{aligned}
$$

This implies the assertion.

We now want to show that $V$ diagonalizes the operator, i.e. $V\left(A^{j} u\right)(\lambda)=\lambda^{j} V(u)(\lambda)$ for all $u \in D\left(A^{j}\right)$. In order to formulate this precisely for a measurable function $\psi: \mathbb{R} \rightarrow \mathbb{R}$ we denote the corresponding multiplication operator on $L_{q}^{2}$ by $M_{\psi}$, i.e. for a function $F \in L_{q}^{2}$ we have

$$
\left(M_{\psi} F\right)_{k}(\lambda)=\psi(\lambda) F_{k}(\lambda), \quad \lambda \in\left[a_{k},+\infty\right), k \in\{1, \ldots, n\} .
$$

Then we have the following lemma, whose proof is again analogous to that of [2, Lemma 4.12].

Lemma 7.7. $\quad$ i) Let $j \in \mathbb{N}$ and $p_{j}: \mathbb{R} \rightarrow \mathbb{R}$ be defined by $p_{j}(x)=x^{j}$. Then for any $f \in D\left(A^{j}\right)$ we have

$$
V\left(A^{j} f\right)=M_{p_{j}} V f .
$$


ii) Let $\psi:\left[a_{1},+\infty\right) \rightarrow \mathbb{R}$ be a bounded, measurable function. Then $\psi(A)$ defined by the spectral Theorem is a bounded operator on $H$ and for all $f \in H$ we have

$$
V(\psi(A) f)=M_{\psi}(V f) \text {. }
$$

In order to show injectivity of $Z$, and thus the surjectivity of $V$, we will need the following property of the kernel.

Lemma 7.8. Let $G \in \operatorname{ker}(Z)$ and let $\psi:\left[a_{1},+\infty\right)$ be measurable and bounded. Then $M_{\psi} G \in$ $\operatorname{ker}(Z)$.

Proof. Due to the boundedness of $\psi$, it holds $D(\psi(A))=H$ and $M_{\psi} G \in L_{q}^{2}$ for $G \in L_{q}^{2}$. Therefore, if $f \in L^{2}(N)$ with compact support and $G \in \operatorname{ker}(Z)$, we have using (20) and Lemma 7.7

$$
\begin{aligned}
\left(Z\left(M_{\psi} G\right), f\right)_{H} & =\left(M_{\psi} G, V f\right)_{L_{q}^{2}}=\left(G, M_{\psi} V f\right)_{L_{q}^{2}}=(G, V \psi(A) f)_{L_{q}^{2}} \\
& =(Z(G), \psi(A) f)_{H}=(0, \psi(A) f)_{H}=0 .
\end{aligned}
$$

This lemma allows to reduce the proof of the injectivity for $Z$ to the frequency bands $\left(a_{k}, a_{k+1}\right)$ for $k=1, \ldots, n$ (set again $a_{n+1}=+\infty$ ). There the injectivity of the Fourier sine transform will be used.

Theorem 7.9. $\operatorname{ker}(Z)=\{0\}$ and thus $V$ is a spectral representation of $H$ relative to $A$.

Proof. Let $G \in L_{q}^{2}$ with $Z(G)=0$ be given. We fix $j \in\{1, \ldots, n\}$ and consider a compact interval $I \subseteq\left(a_{j}, a_{j+1}\right)$. Since $\mathbf{1}_{I} G$ lies in $Y$, invoking Lemmas 7.8 and 7.6 , we obtain for almost all $x \in N$

$$
0=Z\left(\mathbf{1}_{I} G\right)(x)=\sum_{k=1}^{n} \int_{\left(a_{k},+\infty\right)} q_{k}(\lambda) \mathbf{1}_{I}(\lambda) G_{k}(\lambda) F_{\lambda}^{-, k}(x) d \lambda=\sum_{k=1}^{j} \int_{I} q_{k}(\lambda) G_{k}(\lambda) F_{\lambda}^{-, k}(x) d \lambda .
$$

In this equation, we now let $x$ to zero. This is possible, since the hypotheses of Lebesgue's Theorem are satisfied thanks to the presence of $\mathbf{1}_{I}$. This implies thanks to $F_{\lambda}^{-, k}(0)=1$

$$
0=\int_{I} \sum_{k=1}^{j} q_{k}(\lambda) G_{k}(\lambda) d \lambda
$$

Since this is true for every compact interval $I \subseteq\left(a_{j}, a_{j+1}\right)$ and since $q_{k} G_{k} \in L_{\text {loc }}^{1}\left(\left(a_{j}, a_{j+1}\right)\right)$ for all $k=1, \ldots, j$, we can argue as in the proof of Lemma 5.1 to obtain

$$
\sum_{k=1}^{j} q_{k}(\lambda) G_{k}(\lambda)=0 \quad \text { for a.a. } \lambda \in\left(a_{j}, a_{j+1}\right) .
$$

In a second step, we now consider $x \in N_{\ell}$ for some $\ell \leq j$ in (21). This leads to

$$
\begin{aligned}
0 & =\sum_{\substack{k=1 \\
k \neq \ell}}^{j} \int_{I} q_{k}(\lambda) G_{k}(\lambda) \exp \left(-i \xi_{\ell}(\lambda) x\right) d \lambda+\int_{I} q_{\ell}(\lambda) G_{\ell}(\lambda)\left[\cos \left(\xi_{\ell}(\lambda) x\right)-i s_{\ell} \sin \left(\xi_{\ell}(\lambda) x\right)\right] d \lambda \\
& =\sum_{k=1}^{j} \int_{I} q_{k}(\lambda) G_{k}(\lambda) \exp \left(-i \xi_{\ell}(\lambda) x\right) d \lambda-i \int_{I} q_{\ell}(\lambda) G_{\ell}(\lambda)\left(s_{\ell}(\lambda)-1\right) \sin \left(\xi_{\ell}(\lambda) x\right) d \lambda .
\end{aligned}
$$

Thanks to (22), the sum in the last expression vanishes, so we are left with

$$
0=\int_{I} q_{\ell}(\lambda) G_{\ell}(\lambda)\left(s_{\ell}(\lambda)-1\right) \sin \left(\xi_{\ell}(\lambda) x\right) d \lambda .
$$


In this case $\xi_{\ell}(\lambda)=\sqrt{\left(\lambda-a_{\ell}\right) / c_{\ell}}$ is real, so we may substitute $p=\xi_{\ell}(\lambda)$ and obtain

$$
\begin{aligned}
0 & =2 c_{\ell} \int_{\xi_{\ell}(I)} q_{\ell}\left(a_{\ell}+c_{\ell} p^{2}\right) G_{\ell}\left(a_{\ell}+c_{\ell} p^{2}\right)\left[s_{\ell}\left(a_{\ell}+c_{\ell} p^{2}\right)-1\right] \sin (p x) p d p \\
& =2 c_{\ell} \int_{(0,+\infty)} \mathbf{1}_{\xi_{\ell}(I)}(p) q_{\ell}\left(\xi_{\ell}^{-1}(p)\right) G_{\ell}\left(\xi_{\ell}^{-1}(p)\right)\left[s_{\ell}\left(\xi_{\ell}^{-1}(p)\right)-1\right] p \sin (p x) d p .
\end{aligned}
$$

This last integral is now the Fourier sine transform of $\mathbf{1}_{\xi_{\ell}(I)}\left(q_{\ell} \circ \xi_{\ell}^{-1}\right)\left(G_{\ell} \circ \xi_{\ell}^{-1}\right)\left[\left(s_{\ell} \circ \xi_{\ell}^{-1}\right)-1\right] \mathrm{id}$, so by the injectivity of this transformation we find

$$
2 c_{\ell} q_{\ell}\left(\xi_{\ell}^{-1}(p)\right) G_{\ell}\left(\xi_{\ell}^{-1}(p)\right)\left[\left(s_{\ell}\left(\xi_{\ell}^{-1}(p)\right)-1\right] p=0 \quad \text { for a.a. } p \in \xi_{\ell}(I)\right.
$$

Dividing by $p$ and resubstituting this implies

$$
2 c_{\ell} q_{\ell}(\lambda) G_{\ell}(\lambda)\left[s_{\ell}(\lambda)-1\right] \xi_{\ell}(\lambda)=0 \quad \text { for a.a. } \lambda \in I .
$$

Observing, that $q_{\ell}, \xi_{\ell}$ and $s_{\ell}-1$ all may only vanish in the points $a_{1}, \ldots, a_{n}$, this finally implies $G_{\ell}(\lambda)=0$ for all $\ell \in\{1, \ldots, j\}$ and almost all $\lambda \in\left(a_{j}, a_{j+1}\right)$. Since $j \in\{1, \ldots, n\}$ was chosen arbitrarily, the assertion follows.

Note that these considerations also provide a proof for the special case $n=2$, whose proof in [2, Theorem 4.17] is not entirely correct.

Finally, we get the following characterization of the spaces $D\left(A^{j}\right)$.

Theorem 7.10. For $j \in \mathbb{N}$ the following statements are equivalent:

i) $u \in D\left(A^{j}\right)$,

ii) $\lambda \mapsto \lambda^{j}(V u)(\lambda) \in L_{q}^{2}$,

iii) $\lambda \mapsto \lambda^{j}(V u)_{k}(\lambda) \in L^{2}\left(\left(a_{k},+\infty\right), q_{k}\right)$, for all $k=1, \ldots, n$.

Proof. Due to Theorem [7.5, it holds $\left(A^{j} u, A^{j} u\right)_{H}=\left|V\left(A^{j} u\right)\right|_{q}^{2}$. Now Lemma 7.7 implies

$$
\left(A^{j} u, A^{j} u\right)_{H}=\left|M_{p_{j}} V u\right|_{q}^{2} .
$$

The above results provide explicit solution formulae for evolution equations involving the operator $A$. For example for the wave equation $\ddot{u}+A u=0$ with $u(0)=u_{0}$ and $\dot{u}(0)=0$ a formal solution is given by $u(t)=Z \cos (\sqrt{\lambda} t) V u_{0}$. Our aim in the future will be to study properties of these solutions, as indicated in the introduction.

\section{Appendix}

The book of J. Weidmann [27] describes a general approach to the spectral theory of systems of Sturm-Liouville equations, which is in principle applicable to our setting. In this point of view, our problem is seen as a system of $n$ equations on $(0,+\infty)$ coupled by boundary conditions in 0 . Thus the kernel of the resolvent is a matrix-valued function $\mathcal{K}(\cdot, \cdot, \lambda):(0,+\infty) \times(0,+\infty) \rightarrow$ $\mathbb{C}^{n \times n}$. The relation to Definition 3.6 is given by

$$
\mathcal{K}_{i j}\left(x, x^{\prime}, \lambda\right)=K\left(x, x^{\prime}, \lambda\right) \text { where }\left(x, x^{\prime}\right) \in N_{i} \times N_{j} \widehat{=}(0,+\infty) \times(0,+\infty) .
$$


The fundamental hypothesis of Weidmann is that the kernel of the resolvent can be written in the following form:

$$
\mathcal{K}\left(x, x^{\prime}, \lambda\right)= \begin{cases}\sum_{q=1}^{p} \underbrace{\left(\sum_{l=1}^{p} \alpha_{l q} w_{l}(x, \lambda)\right)}_{=: m_{q}^{\alpha}(x, \lambda)} w_{q}\left(x^{\prime}, \lambda\right)^{T}, & \text { for } x^{\prime} \leq x, \\ \sum_{q=1}^{p} \underbrace{\left(\sum_{l=1}^{p} \beta_{l q} w_{l}(x, \lambda)\right)}_{=: m_{q}^{\beta}(x, \lambda)} w_{q}\left(x^{\prime}, \lambda\right)^{T}, & \text { for } x^{\prime}>x,\end{cases}
$$

where $\alpha_{l q}, \beta_{l q} \in \mathbb{C}$ and the $w_{q}:[0, \infty) \times \mathbb{C} \rightarrow \mathbb{C}^{n}$ are such that $\left\{w_{q}(\cdot, \lambda): q=1, \ldots, p\right\}$ is a fundamental system of $\operatorname{ker}\left(A_{f}-\lambda I\right)$, the space of generalized eigenfunctions of $A$. Here $A_{f}: D\left(A_{f}\right) \rightarrow C^{0}(\mathbb{R})$ is the formal operator, in our case $A_{f}=A=\left(-c_{k} \cdot \partial_{x}^{2}+a_{k}\right)_{k=1, \ldots, n}$ but $D\left(A_{f}\right)=\prod_{k=1}^{n} C^{2}\left(N_{k}\right)$, i.e. the operator $A$ without transmission conditions nor integrability conditions at $\infty$. Clearly in our case $\operatorname{dim}\left(\operatorname{ker}\left(A_{f}-\lambda I\right)\right)=2 n$ and thus $p=2 n$.

In contrast to the typical applications treated in [27] as for instance the Dirac system, we consider in this paper a transmission problem, i.e. intuitively the components of the $w_{q}$ are functions on different domains $N_{k}$ (while mathematically all $N_{k}$ are equivalent to $(0,+\infty)$ ): the branches of a star. For all such applications it is highly important to use only generalized eigenfunctions satisfying the transmission conditions $\left(T_{0}\right)$ and $\left(T_{1}\right)$, for example Theorem 7.10 would be impossible otherwise.

Supposing the ansatz (23) of Weidmann, this is not possible, what we shall show now.

In Definition 3.6 we have given an explicit expression for the (unique) kernel $K$ of the resolvent of $A$, using only elements of $\operatorname{ker}\left(A_{T}-\lambda I\right)$, where $A_{T}: D\left(A_{T}\right) \rightarrow H$ satisfies $A_{T}=A=\left(-c_{k} \cdot \partial_{x}^{2}+\right.$ $\left.a_{k}\right)_{k=1, \ldots, n}$ and $D\left(A_{T}\right)=\prod_{k=1}^{n} C^{2}\left(N_{k}\right) \cap\left\{\left(u_{k}\right)_{k=1}^{n}\right.$ satisfies $\left.\left(T_{0}\right),\left(T_{1}\right)\right\}$. Note that $\operatorname{ker}\left(A_{T}-\lambda I\right)$ is the $n$-dimensional space of generalized eigenfunctions of $A$ satisfying the transmission conditions $\left(T_{0}\right)$ and $\left(T_{1}\right)$, but without integrability conditions at infinity.

Suppose that we have a representation of $K$ as given in (23) satisfying

$$
w_{q}(\cdot, \lambda) \in \operatorname{ker}\left(A_{T}-\lambda I\right), q=1, \ldots, p, \lambda \in \rho(A)
$$

and thus we can take $p=n$. Let us fix $x \in N_{1}$ and $\lambda \in \rho(A)$. Then the $m_{q}^{\beta}(x, \lambda)=: m_{q}^{\beta} \in \mathbb{C}$ are constants and the expression

$$
g\left(x^{\prime}, \lambda\right):=\sum_{q=1}^{p} m_{q}^{\beta} w_{q}\left(x^{\prime}, \lambda\right)
$$

defines a function

Clearly, the functions

$$
g(\cdot, \lambda):\left\{x^{\prime} \in[0, \infty): x^{\prime}>x\right\} \rightarrow \mathbb{C}^{n} .
$$

$$
\left[w_{q}(\cdot, \lambda)\right]_{j}: N_{j} \rightarrow \mathbb{C}
$$

can be uniquely extended to entire functions in $x^{\prime}$ because they are linear combinations of $e^{ \pm i \xi_{j}(\lambda) x^{\prime}}$. Thus the $[g(\cdot, \lambda)]_{j}$ are entire. Our hypothesis $w_{q}(\cdot, \lambda) \in \operatorname{ker}\left(A_{T}-\lambda I\right), q=1, \ldots, p$, implies thus

$$
g(\cdot, \lambda) \in \operatorname{ker}\left(A_{T}-\lambda I\right), q=1, \ldots, p .
$$

But comparing (23) with Definition 3.6 yields

$$
g\left(x^{\prime}, \lambda\right)=F_{\lambda}^{ \pm, 2}(x) \cdot F_{\lambda}^{ \pm, 1}\left(x^{\prime}\right) \text { for } x^{\prime} \in N_{2}, \ldots, N_{n}
$$


and

$$
g\left(x^{\prime}, \lambda\right)=F_{\lambda}^{ \pm, 1}(x) \cdot F_{\lambda}^{ \pm, 2}\left(x^{\prime}\right) \text { for } x^{\prime} \in N_{1},
$$

which makes sense after analytic continuation. Using the assumption that $g$ satisfies $\left(T_{0}\right)$, we get from these two equalities, putting $x^{\prime}=0 \in \overline{N_{2}}$ into the first and $x^{\prime}=0 \in \overline{N_{1}}$ into the second

$$
F_{\lambda}^{ \pm, 2}(x)=F_{\lambda}^{ \pm, 2}(x) F_{\lambda}^{ \pm, 1}(0)=g(0, \lambda)=F_{\lambda}^{ \pm, 1}(x) F_{\lambda}^{ \pm, 2}(0)=F_{\lambda}^{ \pm, 1}(x) .
$$

But inspecting the definitions of the generalized eigenfunctions, bearing in mind that $x \in N_{1}$ was arbitrary, this implies

$$
\cos \left(\xi_{1}(\lambda) x\right) \pm i \sin \left(\xi_{1}(\lambda) x\right)=\cos \left(\xi_{1}(\lambda) x\right) \pm i s_{1}(\lambda) \sin \left(\xi_{1}(\lambda) x\right)
$$

and thus $s_{1}(\lambda)=1$. This finally implies $\sum_{j=1}^{n} c_{j} \xi_{j}(\lambda)=0$, which is impossible for all real $\lambda<a_{1}$, since then $\xi_{j}(\lambda)$ is purely imaginary. Furthermore, $\sum_{j=1}^{n} c_{j} \xi_{j}$ is analytic, so this equality can, if ever, only be fulfilled on a discrete set of $\lambda \in \mathbb{C}$.

We have thus proven:

Theorem 8.1. Representation (23) of the kernel $\mathcal{K}$ of the resolvent of $A$ is not possible, using exclusively generalized eigenfunctions

$$
w_{q}(\cdot, \lambda) \in \operatorname{ker}\left(A_{T}-\lambda I\right), q=1, \ldots, p .
$$

The reason for this is, shortly speaking, the rigidity of (23) caused by the use of the same linear combinations of generalized eigenfunctions above the diagonals of all $N_{j} \times N_{k}$ (idem below). This is not compatible with the fact that the kernel is non-smooth only on the main diagonals, i.e. the diagonals of $N_{j} \times N_{j}$, cf. Definition 3.6 and Figure 1.

This means that the approach of J. Weidmann in [27], when applied to problems on the starshaped domain, does not sufficiently take into account its non-manifold character. The resulting expansion formulae would use generalized eigenfunctions which are in a sense incompatible with the geometry of the domain. This would have undesirable consequences: for example, the important feature of Theorem 7.10 that the belonging of $u$ to $D\left(A^{j}\right)$ is expressed by the decay of the components of $V u$ would be impossible, due to artificial singularities in the expansion formula.

\section{REFERENCES}

[1] F. Ali Mehmeti, Nonlinear Waves in Networks. Mathematical Research, vol. 80, Akademie Verlag, Berlin, 1994.

[2] F. Ali Mehmeti, Spectral Theory and $L^{\infty}$-time Decay Estimates for Klein-Gordon Equations on Two Half Axes with Transmission: the Tunnel Effect. Math. Methods Appl. Sci. 17 (1994), 697-752.

[3] F. Ali Mehmeti, Transient Waves in Semi-Infinite Structures: the Tunnel Effect and the Sommerfeld Problem. Mathematical Research, vol. 91, Akademie Verlag, Berlin, 1996.

[4] F. Ali Mehmeti, J. von Below, S. Nicaise (eds.), Partial differential equations on multistructures. Lecture Notes in Pure and Appl. Math., vol. 219, Marcel Dekker, New York, 2001.

[5] F. Ali Mehmeti, R. Haller-Dintelmann, V. Régnier, Expansions in generalized eigenfunctions of the weighted Laplacian on star-shaped networks. H. Amann, W. Arendt, M. Hieber, F. Neubrander, S. Nicaise, J. von Below (eds): Functional analysis and Evolution Equations; The Günter Lumer Volume, Birkhäuser, Basel, 2008, 1-16.

[6] F. Ali Mehmeti, E. Meister, K. Mihalinčić, Spectral Theory for the Wave Equation in Two Adjacent Wedges. Math. Methods Appl. Sci. 20 (1997), 1015-1044.

[7] F. Ali Mehmeti, V. Régnier, Splitting of energy of dispersive waves in a star-shaped network. Z. Angew. Math. Mech. 83 (2003), no. 2, 105-118.

[8] F. Ali Mehmeti, V. Régnier, Delayed reflection of the energy flow at a potential step for dispersive wave packets. Math. Methods Appl. Sci. 27 (2004), 1145-1195. 
[9] F. Ali Mehmeti, V. Régnier, Global existence and causality for a transmission problem with a repulsive nonlinearity. Nonlinear Anal. 69 (2008), 408-424.

[10] J. von Below, J.A. Lubary, The eigenvalues of the Laplacian on locally finite networks. Results Math. 47 (2005), no. 3-4, 199-225.

[11] J.M. Berezanskii, Expansions in eigenfunctions of selfadjoint operators. Transl. Math. Monogr., vol. 17, American Mathematical Society, Providence, 1968.

[12] S. Cardanobile and D. Mugnolo. Parabolic systems with coupled boundary conditions. J. Differential Equations 247 (2009), no. 4, 1229-1248.

[13] E. Croc, Y. Dermenjian, Analyse spectrale d'une bande acoustique multistratifiée I : principe d'absorption limite pour une stratification simple. SIAM J. Math. Anal. 26 (1995), no. 24, 880-924.

[14] Y. Daikh, Temps de passage de paquets d'ondes de basses fréquences ou limités en bandes de fréquences par une barrière de potentiel. Thèse de doctorat, Valenciennes, France, 2004.

[15] J.M. Deutch, F.E. Low, Barrier Penetration and Superluminal Velocity. Annals of Physics 228 (1993), 184202.

[16] B. Dorn, Semigroups for flows in infinite networks. Semigroup Forum 76 (2008), no. 2, 341-356.

[17] N. Dunford, J.T. Schwartz, Linear Operators II. Wiley Interscience, New York, 1963.

[18] A. Enders, G. Nimtz, On superluminal barrier traversal. J. Phys. I France 2 (1992), 1693-1698.

[19] P. Exner, J.P. Keating, P. Kuchment, T. Sunada, A. Teplyaev (eds.), Analysis on Graphs and Its Applications. Proc. Sympos. Pure Math., vol. 77, AMS, 2008.

[20] A. Haibel, G. Nimtz, Universal relationship of time and frequency in photonic tunnelling. Ann. Physik (Leipzig) 10 (2001), 707-712.

[21] G. Heinzelmann, P. Werner, Resonance phenomena in compound cylindrical waveguides. Math. Methods Appl. Sci. 29 (2006), no. 8, 877-945.

[22] V. Kostrykin, R. Schrader, The inverse scattering problem for metric graphs and the travelling salesman problem. Preprint, 2006 (www.arXiv.org math.AP/0603010).

[23] M. Kramar, E. Sikolya, Spectral properties and asymptotic periodicity of flows in networks. Math. Z. 249 (2005), 139-162.

[24] T. Poerschke, G. Stolz, J. Weidmann, Expansions in Generalized Eigenfunctions of Selfadjoint Operators. Math. Z. 202 (1989), 397-408.

[25] M. Pozar, Microwave Engineering. Addison-Wesley, New York, 1990.

[26] W. Rudin, Real and Complex Analysis. McGraw-Hill Book Co., New York-Toronto, Ont.-London, 1966.

[27] J. Weidmann, Spectral Theory of Ordinary Differential Operators. Lecture Notes in Mathematics, vol. 1258, Springer-Verlag, Berlin, 1987.

Univ Lille Nord de France, F-59000 Lille, France

UVHC, LAMAV, FR CNRS 2956, F-59313 VAlenciennes, France

E-mail address: felix.ali-mehmeti@univ-valenciennes.fr

TU Darmstadt, Fachbereich Mathematik, Schlossgartenstrasse 7, 64289 Darmstadt, Germany E-mail address: haller@mathematik.tu-darmstadt.de

Univ Lille Nord de France, F-59000 Lille, France

UVHC, LAMAV, FR CNRS 2956, F-59313 VALENCIENNES, France

E-mail address: Virginie.Regnier@univ-valenciennes.fr 